\title{
Space, object, and task selection
}

\author{
Joel LACHTER \\ San Jose State University, San Jose, California \\ and NASA Ames Research Center, Moffett Field, California \\ ROGER W. REMINGTON \\ University of Queensland, St. Lucia, Queensland, Australia \\ AND \\ ERIC RUTHRUfF \\ University of New Mexico, Albuquerque, New Mexico
}

\begin{abstract}
Within the field of selective attention, two separate literatures have developed, one examining the effect of selection of objects and another examining the effect of selection of features. The present study bridged these two traditions by examining the compatibility effects generated by two features of attended and unattended nontarget (foil) stimuli. On each trial, participants determined either the identity or the orientation of a visual stimulus. Spatial attention was controlled using cues (presented prior to the target frame) designed to involuntarily capture attention. We independently manipulated the stimulus dimension the participants prepared for and the stimulus dimension on which they actually executed the task. Preparation had little influence on the magnitude of compatibility effects from foil stimuli. For attended stimuli, the stimulus dimension used in executing the task produced large compatibility effects, regardless of whether that dimension was prepared. These and other compatibility effects (e.g., Stroop effects) are discussed in relation to an integrative model of attentional selection. The key assumptions are that (1) selection occurs at three distinct levels (space, object, and task), (2) spatial attention leads to semantic processing of all dimensions, and (3) features do not automatically activate responses unless that object is selected for action.
\end{abstract}

Selective attention, by definition, involves the preferential processing of certain stimuli over others. Understanding the effects of such selection on stimulus processing has been a major goal of experimental psychology. Interestingly, two separate literatures have developed, one examining the effect of selection of objects (following James [1890], Broadbent [1958], and Posner [1980]) and another examining the effect of selection of features (following Stroop [1935] and Garner [1974]). Recently, a few researchers have attempted to bridge the gap between these literatures (Maruff, Danckert, Camplin, \& Currie, 1999; Mounts \& Melara, 1999; Remington \& Folk, 2001; Shalev \& Algom, 2000). Here, we will present new evidence that selection of features occurs after selection of objects. We then will present a model capable of explaining a wide range of seemingly contradictory effects concerning the selection of objects and features.

There is general agreement that the selection of objects occurs at multiple levels of processing, including both early perceptual stages and late response stages (e.g., Dark, Johnston, Myles-Worsley, \& Farah, 1985; Desi- mone \& Duncan, 1995; Johnston, McCann, \& Remington, 1995). For example, studies of spatial attention have shown that attended objects are processed semantically but unattended objects often are not (e.g., Besner, Risko, \& Sklair, 2005; Lachter, Forster, \& Ruthruff, 2004; McCann, Folk, \& Johnston, 1992; Vogel, Woodman, \& Luck, 2005; Yantis \& Johnston, 1990; but see also Brown, Gore, \& Carr, 2002; Lachter, Ruthruff, Lien, \& McCann, 2008). Objects that are selected at this early spatial attention level may still fail to be selected at more central stages of processing (Allport, Tipper, \& Chmiel, 1985).

A limitation of these studies is that they generally have treated the entire object as the unit of selection. In the real world, people often must identify and react to objects on the basis of a single dimension, independently of other dimensions (e.g., picking a sock on the basis of its color, rather than on the basis of its orientation, location, or shape). Other lines of research have examined the selection of specific features of objects (the Stroop paradigm - Stroop, 1935; MacLeod, 1991-discussed below, is a prominent example). However, this literature largely

J. Lachter, joel.b.lachter@nasa.gov 
has ignored the need for selecting the target from among possible nontarget objects (see Shalev \& Algom, 2000). ${ }^{1}$

\section{Implications of Object Selection for Feature Selection}

Although there is considerable research on spatial selection of entire objects and selection of specific features, very little is known about how these forms of selection interact. Some have argued that focusing spatial attention on an object facilitates processing of all the dimensions of that object (e.g., color, size, and shape; Duncan, 1984; Kahneman \& Chajczyk, 1983), whereas others have argued that attention facilitates only those features that match a specific task set (Maruff et al., 1999; Mounts \& Melara, 1999; Remington \& Folk, 2001).

Initial evidence that people identify all the dimensions of an attended target, even ones irrelevant to the current task, came from the well-known Stroop effect (Stroop, 1935; MacLeod, 1991). Stroop discovered that people have great difficulty naming the ink color of a word that happens to spell the name of a conflicting color (e.g., the word GREEN in red ink). This finding implies that people identify words even when they are irrelevant. Thus, some irrelevant dimensions of attended stimuli appear to be processed sufficiently to be identified.

Duncan (1984) introduced a different way of investigating the processing of multiple dimensions of attended stimuli. Rather than asking whether stimulus dimensions are processed even when irrelevant, he asked whether there is a cost of processing multiple relevant dimensions at the same time. He presented people with two overlapping objects (with two dimensions each) and asked them to identify one or two specific properties. The requirement to process two features from different objects produced a large decrement in performance (relative to identifying a single feature). The requirement to process two features of the same object, however, produced very little decrement in performance. This study showed that people can process multiple relevant dimensions of one object about as well as they can process a single dimension. These findings are consistent with the hypothesis that attention to an object causes all of its dimensions to be processed fully.

More recent studies have challenged the claim that all the features of an attended object are accessed (Cohen \& Shoup, 1997; Maruff et al., 1999; Mounts \& Melara, 1999; Remington \& Folk, 2001). These studies have examined compatibility effects ${ }^{2}$ on target response times (RTs) from irrelevant stimuli that have features associated with one response or the other. We refer to such stimuli as foils. Cohen and Shoup (1997), for example, examined compatibility effects between a central target and two flanking items that were not relevant to the task (foils). Their stimuli were bars, which could be colored red, green, or blue and could be oriented vertically, slanted left, or slanted right. Participants were to press one key if the target was red or slanted right and another key if the target was green or slanted left. Blue and vertical were designated as neutral values (not associated with any response). The participants were not told in advance which dimension (color or orientation) would be relevant, but on each trial, only one of the dimensions had a value that was assigned to a response (the other dimension had a neutral value). Because the participants could not selectively prepare in advance for only the appropriate dimension, they presumably prepared for both dimensions (or selected one dimension at random).

In this design, there are two dimensions of the foil that might produce a compatibility effect on responses to the central target: the relevant dimension and the irrelevant dimension. Here, we will refer to a foil dimension as being relevant if it is the dimension used to execute the target task (even though the value of the foil on that relevant dimension is, of course, uninformative). For example, responses to the target's color could be influenced by both the foil's color (the relevant dimension) and its slant (the irrelevant dimension). Across several experiments, Cohen and Shoup (1997) found large effects of the target's compatibility with the relevant dimension of the foil, but not with the irrelevant dimension of the foil. They argued that an early processing stage activates responses for each dimension separately; then a later stage selects responses on the basis of dimension relevance. Cohen and Shoup's (1997) method has been challenged on the grounds that some features were presented more often than others (Mordkoff, 1998). However, their central finding (the irrelevant dimension of the foil produces little or no compatibility effect) has since been replicated under conditions in which all the features occurred equally often (Maruff et al., 1999; Remington \& Folk, 2001).

A drawback of the flanker paradigm used by Cohen and Shoup (1997) is that the spatial focus of attention is not controlled, making it unclear which objects were attended. In a similar study, Remington and Folk (2001) found no compatibility effect (even from the relevant dimension of the foil) when the foil was unattended. Remington and Folk achieved control over the locus of spatial attention by using a variant of the search paradigm with two possible tasks (letter identity and orientation). Targets could appear in one of four possible locations marked with boxes. Before each trial, participants were told to respond to either the identity ( $T$ or $L$ ) or orientation (slanted left or slanted right) of the red target stimulus, whose location was not known in advance. Next, an uninformative exogenous cue (four red dots), thought to reliably capture attention (Folk, Remington, \& Johnston, 1992), was presented surrounding one of the four locations. Next, the red target and three white distractors appeared briefly. Two of the three white distractors were neutral stimuli (not associated with a response), but one (the foil) was a slanted $L$ or $T$. This design allowed Remington and Folk to measure the influence of attention on compatibility effects from three different stimulus dimensions: (1) the relevant dimension of the foil, (2) the irrelevant dimension of the foil, and (3) the irrelevant dimension of the target.

When the foil was not in the cued location (i.e., not attended), neither the relevant nor the irrelevant dimension produced a compatibility effect. When the foil was in the cued location (i.e., was attended), however, Rem- 
ington and Folk (2001) found large compatibility effects for the relevant foil feature but no compatibility effect for the irrelevant foil feature. Although the irrelevant foil feature had no effect, even when the foil was attended, the irrelevant target feature had a large effect. This intriguing pattern of results was replicated in a second experiment in which the target was defined by motion, rather than by color, and the task was to respond to either color or orientation.

Similar findings have been found using a negativepriming paradigm (Fanini, Nobre, \& Chelazzi, 2006; Frings \& Wentura, 2006; Tipper, Weaver, \& Houghton, 1994). The key finding in this paradigm is that responses to a target are especially slow if that object or feature had to be ignored in the previous trial. This finding supports the hypothesis that representations of to-be-ignored items are suppressed in some way, making them relatively inaccessible in the near future. In a pattern similar to that found by Remington and Folk (2001), both Tipper et al. and Frings and Wentura found that the relevant feature of an ignored object consistently produced negative priming on the next trial but the irrelevant feature did not. Furthermore, Fanini et al. found that an irrelevant feature of a target did produce negative priming on a subsequent trial. Although these findings are generally consistent with those of Remington and Folk, the results depended somewhat on the particular judgments being studied. For example, in the Fanini et al. study, participants had to judge either the color or the motion of a target. When they judged color, responses to a subsequent target with the same motion were delayed. However, if they judged motion, no negative priming effect was observed for subsequent targets with the same color. Such differences are presumably due to the fact that negative priming requires some sort of episodic retrieval of the previous trial (Tipper, 2001). Differences between features and other aspects of the experimental context may affect the degree to which one trial evokes such an episodic memory trace of the previous trial (Park \& Kanwisher, 1994).

\section{Attention Versus Selection}

Remington and Folk's (2001) findings, and those in the negative-priming literature, present a challenge to any theory of selective attention: How is it that the irrelevant dimension of a target affects responses but the irrelevant dimension of an attended foil does not? Both objects were attended, so one might presume that they would produce qualitatively similar patterns of compatibility effects. As Remington and Folk noted, these findings suggest a critical (but often ignored) distinction between spatially attending a location/object (which occurs for both targets and foils) and actually selecting an object for action (which occurs only for targets). Although they pointed out the importance of this distinction, they did not specify exactly how attended and selected objects are treated differently. In this article, we will explore two different possibilities, discussed below.

We will refer to the first possible account of how attention and selection differ as the preparation account. This account assumes that attention does not necessarily lead to the identification of all the features of an attended object. If participants know in advance of each trial which dimension will be relevant (as was the case in Remington \& Folk, 2001), they can filter out the irrelevant dimension prior to identification. Thus, when the cue draws attention to the foil, it is the prepared for (unfiltered) dimension that is processed most deeply (producing compatibility effects) before attention moves on to the target. By itself, however, this hypothesis fails to explain why the unprepared/ irrelevant dimension of the target still managed to strongly influence responses. To explain this finding, one could further assume that the subsequent selection of the target object for response triggers the allocation of processing resources to all the dimensions (prepared for and unprepared for) of that object. According to this account of Remington and Folk's results, compatibility effects are produced primarily by foil dimensions to which participants prepare to respond. This preparation account predicts that if participants prepare for both tasks (e.g., because they do not know in advance which target task will be relevant), compatibility effects should be just as large for irrelevant foil dimensions as for relevant foil dimensions.

An alternative to the preparation account has been proposed by Cohen and Shoup $(1997,2000)$. According to this account, features from different dimensions are processed in separate modules until the point at which features are conjoined to make objects (Treisman \& Gelade, 1980). The relevant foil feature is processed within the same module as the relevant target feature, so it can produce a strong compatibility effect. The irrelevant foil feature, however, is not processed within the same module as the relevant target feature and, thus, should not produce a compatibility effect. According to Cohen and Shoup (2000), entire selected objects are passed to response mechanisms, allowing irrelevant features of the selected target object to influence responses. In summary, this account of Remington and Folk's (2001) results asserts that compatibility effects from a foil are produced primarily by the dimension that is ultimately executed. Accordingly, we will refer to this as the execution account. Note that, in this model, there is no need to assume a significant role of preparation in the production of compatibility effects. This account assumes that all dimensions of an attended object are identified, even dimensions that are not prepared for.

\section{The Present Study}

Remington and Folk (2001) did not independently manipulate which dimension was prepared for and which was executed. Consequently, it is unclear which factor (preparation or execution) was responsible for the presence of large compatibility effects with the relevant foil dimension (which was both prepared for and executed) and the absence of compatibility effects from the irrelevant foil dimension (which was neither prepared for nor executed). Below, we will present data from several new experiments in which we pitted these factors against each other.

In Experiment 1, we modified the Remington and Folk (2001) paradigm so that the target task (letter identity vs. 
orientation) was not known in advance. Thus, participants could not systematically prepare for the dimension to which they eventually responded. In Experiments 2-4, preparation and execution were manipulated independently. Specifically, participants knew which task was most likely to be relevant - thus encouraging them to prepare for the associated stimulus dimension - but did not know for sure until after they had identified the target. For example, sometimes, when the participants expected to respond to letter identity, the actual target was a slanted bar rather than a letter (i.e., affording only the orientation judgment). Likewise, sometimes, when the participants expected to respond to slant, the actual target was an upright letter with no slant (i.e., affording only the identity judgment). Each dimension was prepared-for-executed, prepared-for-unexecuted, unprepared-for-executed, or unprepared-for-unexecuted.

Both the preparation and execution accounts predict that we should replicate the main findings of Remington and Folk (2001): a large effect of compatibility with the prepared-for-executed dimension and little or no effect of compatibility with the unprepared-for-unexecuted dimension. As will be discussed below, however, the preparation and execution accounts differ in their predictions for the prepared-for-unexecuted and unprepared-for-executed conditions.

\section{GENERAL METHOD}

The four experiments presented in this article employed closely related methods (similar to those in Remington \& Folk, 2001; see Figure 1):

- Participants were to determine either the orientation or the identity of a red target stimulus, which was presented simultaneously with three white distractor stimuli. The target location was not known in advance.

- Two distractors in each display were neutral letters or symbols, not associated with any response.
- The third distractor (the foil) had at least one feature assigned to a response, potentially giving rise to compatibility effects (Eriksen $\&$ Hoffman, 1973). The conditions under which such compatibility effects would occur (e.g., with or without spatial attention) was the primary empirical issue addressed here.

- A cue consisting of four red dots appeared at one of the four stimulus locations shortly before the onset of the target display. Contingent capture theory (Folk et al., 1992) argues that, because participants differentiate the target from the foil and distractors by its red color, these red dots should automatically attract attention to their location. The results of Experiments 1 and 2, presented below, bear this assumption out.

The exact timing and spacing of the stimuli, shown in Figure 1, were constant across experiments. Four boxes, surrounding the potential stimulus locations, were continuously present on the screen. Trials began with a fixation cross in the center of the screen. After $1 \mathrm{sec}$, the fixation cross disappeared for $51 \mathrm{msec}$ and then reappeared for a variable foreperiod (51-408 $\mathrm{msec}$, in multiples of $51 \mathrm{msec}$ ), then disappeared again. Simultaneous with its disappearance, four small dots surrounding each of these boxes were flashed briefly $(102 \mathrm{msec})$. The dots surrounding three of the boxes were white, whereas those surrounding the fourth were red (the cue). These dots then disappeared, and the screen was blank (except for the four boxes) for $85 \mathrm{msec}$. Following this blank period, four characters appeared for $102 \mathrm{msec}$, one in each box. Two of these characters were neutral distractors, one was the foil, and one was the target. The target was signified by being colored red, whereas the other three characters were white.

If the participants did not respond to the target within $5 \mathrm{sec}$ of its onset, the message "Timed Out" was displayed in red. When the participants made an error, the computer beeped, and the message "Incorrect Response" appeared in red for $2 \mathrm{sec}$. These error trials were repeated later in the session. Feedback on the error rate and average RT was provided at the end of each block.

The primary purpose of these experiments was to determine which features of the foil would be identified. Our evidence for such identification came from the compatibility effect - the degree to which performance was better when a particular foil dimension and the target were assigned to the same response, rather than to different responses. As a result, we focused our analyses on these compatibility effects. Furthermore, since the orientation and letter dimensions of the foil generally have similar effects, we collapsed across task

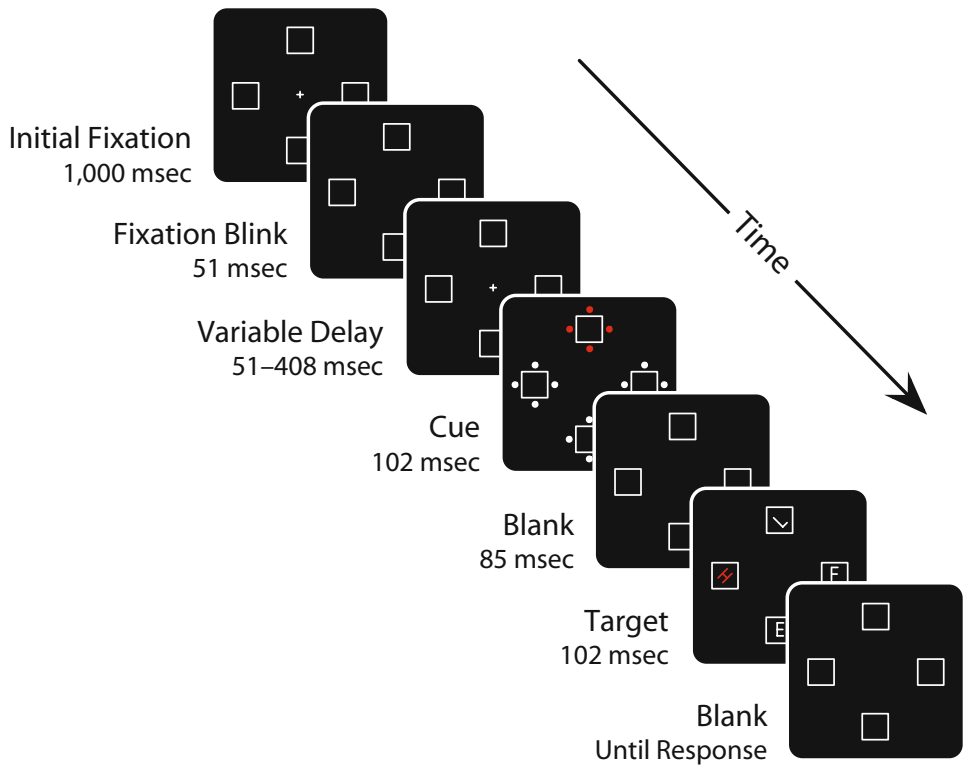

Figure 1. A typical event sequence in Experiments 1-3. 
type (orientation and letter) in these analyses. This allowed for a greatly simplified presentation of the results. A more complete set of analyses is presented as an Appendix.

\section{EXPERIMENT 1}

The purpose of Experiment 1 was to test predictions of our two main hypotheses regarding the effects of task preparation. Before each trial, Remington and Folk (2001) provided participants with a perfectly reliable task cue, which told them which dimension (identity or orientation) would be relevant. The participants needed to prepare selectively for this dimension, because the stimulus itself (a $T$ or $L$ slanted left or right) was always associated with both tasks (dual-affordance stimuli; Ruthruff, Remington, \& Johnston, 2001). In the present experiment, however, we provided no task cue in advance, so that the participants presumably prepared for each dimension to a similar degree. The target stimulus that appeared (an upright $T$ or $L$, or a left/right-slanted $H$ ) was associated with only one of the tasks (single-affordance), indicating that the participants were to perform that task. Thus, it was the target itself, rather than an advance cue, that informed the participants which task to perform.

The main question in this experiment was whether compatibility effects would be strong for both dimensions of the foil (since they should be equally prepared for) or primarily for the executed foil dimension. If foil-target compatibility effects occurred primarily for the executed dimension, this outcome would weigh against a preparation account.

\section{Method}

Participants. Forty students from local colleges participated for pay or course credit. No participants made more than our cutoff criterion of $10 \%$ errors; however, 2 participants were removed because their overall mean RTs were more than three standard deviations $(S D$ s) above the mean for all 40 participants.

Stimuli and Procedure. The general method outlined above was followed. In this experiment, the target could be an $H$ slanted $45^{\circ}$ to the right or left or an upright $T$ or $L$. If the target was a slanted $H$, the participants were to perform the orientation judgment: press the "." key for left-slanting $H$ s and the "/" key for right-slanting $H$ s. If the target was upright, the participants were to perform the letter identity task: press the "." key for Ts and the "/" key for $L$ s. Foils could be either $T$ or $L$ and were slanted $45^{\circ}$ to either the right or the left. Thus, whereas the target afforded only one of the two tasks (orientation or identity), the foil afforded both tasks. The location of the cue, foil, and target were randomized, with the constraint that, across trials, the stimulus appearing in the cued location was the target on $25 \%$ of the trials, the foil on $25 \%$ of the trials, and one of the two distractors on $50 \%$ of the trials. Trials on which the participant made an error were repeated later in the session.

Trials were grouped into nine blocks to provide the participants with breaks (the blocking of trials did not constrain the counterbalancing of conditions). The first block was practice. Each block contained 2 warm-up trials and 64 experimental trials, plus a trial for each error the participant made in that block.

\section{Results}

First, note that the manipulation of spatial attention by varying the cue location appears to have been successful. RTs averaged $576 \mathrm{msec}$ when the target location was cued, $621 \mathrm{msec}$ when a neutral item was cued, and $638 \mathrm{msec}$ when the foil was cued, a statistically significant effect $[F(2,74)=241.17, p<.001]$. Separate analyses showed that RTs were shorter when the target was cued than when a neutral item was cued $[F(1,37)=294.45, p<.001]$ and when a neutral item was cued than when the foil was cued $[F(1,37)=40.14, p<.001]$. The former effect presumably reflects an attention shift back to the target when a nontarget was cued, whereas the latter effect presumably reflects the fact that the foil was not as easily rejected as a nontarget.

The compatibility effect for each combination of cue location (foil, neutral, or target) and execution (executed or unexecuted foil dimension) was calculated by subtracting the mean RT on trials in which that dimension of the foil was congruent with the target response from the mean RT on trials in which it was incongruent. The resulting compatibility effects are shown in Figure 2. An ANOVA was conducted to assess the influence of cue location and execution on compatibility effects. Significant main effects were found for both cue location $[F(2,74)=16.27$, $p<.001]$ and execution $[F(1,37)=20.85, p<.001]$. As might be expected, these two factors combined multiplicatively, resulting in a significant interaction $[F(2,74)=$ $5.28, p<.01]$. A large compatibility effect was found for the relevant dimension when the foil was cued $[t(37)=$ $8.24, p<.001]$, and smaller compatibility effects were found for the irrelevant dimension when the foil was cued $[t(37)=2.29, p<.05]$ and for the relevant dimension when a neutral item was cued $[t(37)=3.22, p<.01]$. Thus, unlike in Remington and Folk (2001), it appears that neither our unattended stimuli nor our irrelevant features were completely suppressed. The latter difference might reflect the fact that the participants prepared for both dimensions in the present Experiment 1 but prepared only for the relevant dimension in Folk and Remington. In other words, the data suggest that preparation might play a minor role in producing compatibility effects, albeit a much smaller role than that played by execution.

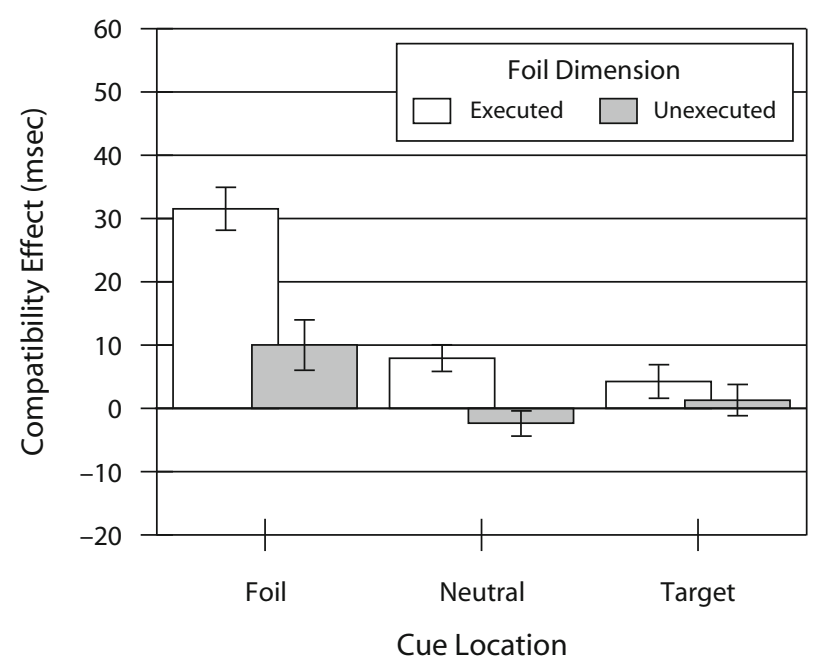

Figure 2. Compatibility effects in Experiment 1. Error bars indicate one standard error. 
The overall error rate was quite low $(<3 \%)$. Nonetheless, a significant main effect of cue location on errors was found $[F(2,74)=7.08, p<.01]$; the participants performed best when the target was cued and worst when the foil was cued. The overall compatibility effect on errors was not different from zero, nor did the compatibility effect depend on cue location or execution.

\section{Discussion}

As in Remington and Folk (2001), the foil dimension corresponding to the task ultimately performed on the target produced large compatibility effects, but the other (nonexecuted) foil dimension produced relatively little effect. Because the participants were equally prepared for both dimensions, this finding cannot have been due to extra advance preparation for the dimension that turned out to be executed on a given trial. Instead, it appears that a key determinant of whether a particular foil dimension will produce a compatibility effect is whether that dimension turns out to be the executed dimension.

\section{EXPERIMENT 2}

On every trial of Experiment 1, each stimulus dimension (letter or orientation) was equally likely to be executed. This fact prevented the participants from systematically preparing in advance for the executed task and, thus, filtering out processing of the unexecuted dimension of the foil. Although the results indicate that compatibility effects were strongest for the executed dimension of the foil, there was some evidence that preparation played a role as well. Furthermore, note that the executed dimension presumably was also prepared for to some degree. Therefore, it remains possible that compatibility effects from a foil dimension depend on some combination of execution and preparation.

To address these possibilities, Experiment 2 selectively manipulated execution and preparation. We manipulated preparation by blocking trials according to which task (orientation or letter identity) was more likely to be executed. Within a block, $75 \%$ of the trials were of the prepared for task type, whereas $25 \%$ of the trials were of the other (unprepared for) task type. Once again, the preparation account predicts that compatibility effects should arise from the dimension to which the participant is prepared to respond, even if it turns out to be unexecuted. Conversely, the execution account predicts effects of the executed dimension, even when unprepared for.

\section{Method}

Participants. Forty-nine students from local colleges participated for pay or course credit. No participants made more than our cutoff criterion of $10 \%$ errors or had a mean RT more than three $S D$ s above the mean for all the participants. However, 3 participants were rejected because they did not contribute data to all the cells of the design.

Stimuli and Procedure. The stimuli were identical to those in Experiment 1 . The procedure differed from that in Experiment 1 only in the way trials were blocked. As in Experiment 1, trials were grouped into nine blocks. The first block, which was practice, included equal numbers of each task (i.e., $50 \%$ orientation and $50 \%$ letter identification). However, the eight remaining blocks were di- vided into four orientation-biased blocks and four identity-biased blocks. In these blocks, the predominant task was presented $75 \%$ of the time (not counting repeats of errors), across all four blocks with the same bias. At the beginning of each block, the participants were told whether the block would be an orientation or a letter identification block. The participants received all four of the blocks biased to one task before any biased toward the other (which task came first was counterbalanced across participants, 23 starting with each task). Each of these blocks contained 2 warm-up trials and 64 experimental trials, plus repeats of any error trials in that block.

\section{Results}

To maximize our ability to detect the effects of preparation, our analyses excluded trials following a trial on which the executed task was unprepared for (note, however, that the same basic pattern of results was obtained when all the trials were included). Our manipulation of attention via cue location was again successful; mean RT was $583 \mathrm{msec}$ when the target location was cued, but $635 \mathrm{msec}$ when a neutral element was cued and $661 \mathrm{msec}$ when the foil was cued $[F(2,90)=183.42, p<.001]$. As in Experiment 1, separate analyses showed that RTs were shorter when the target was cued than when a neutral item was cued $[F(1,45)=167.53, p<.001]$ and when a neutral item was cued than when the foil was cued $[F(1,45)=$ $51.00, p<.001]$. Our manipulation of task preparation via blocking of task bias was also successful; mean RT was $580 \mathrm{msec}$ when the target was of the more likely task type for the block, but $673 \mathrm{msec}$ when it was of the less likely task type $[F(1,45)=194.92, p<.001]$. Thus, it is appropriate to refer to these conditions as prepared for and unprepared for, respectively.

The compatibility effect for each combination of cue location (foil, neutral, or target), preparation (prepared for or unprepared for foil dimension) and execution (executed or unexecuted foil dimension) was calculated by subtracting the average RT on trials on which that dimension of the foil was congruent with the response from that on trials on which it was incongruent. The resulting compatibility effects are shown in Figure 3. We conducted an ANOVA to assess the influence of cue location, preparation, and execution on compatibility effects. As in Experiment 1, significant main effects were found for cue location $[F(2,90)=$ $9.39, p<.001]$ and execution $[F(1,45)=5.27, p<.05]$, as well as their interaction $[F(2,90)=20.36, p<.001]$. Importantly, there was no effect of preparation $[F(1,45)<1]$, nor did preparation interact with cue location $[F(2,90)=2.16]$ or execution $[F(1,45)<1]$. As we noted in the introduction, two conditions were of special interest: the preparedfor-unexecuted and unprepared-for-executed conditions when the foil was cued. Planned $t$ tests were performed to assess these effects. The 2-msec compatibility effect in the prepared-for-unexecuted case was not significant $[t(45)<1]$. However, the 42 -msec compatibility effect in the unprepared-for-executed condition was highly significant $[t(45)=3.86, p<.001]$. The difference between these effects was significant $[t(45)=3.19, p<.005]$.

Again, mean error rates were quite low $(<3 \%)$. Echoing the RT data, the participants were more accurate when the target location was cued ( $2.1 \%$ errors) than when a neutral location was cued (3.2\% errors) or the foil location 
A

Foil Cued

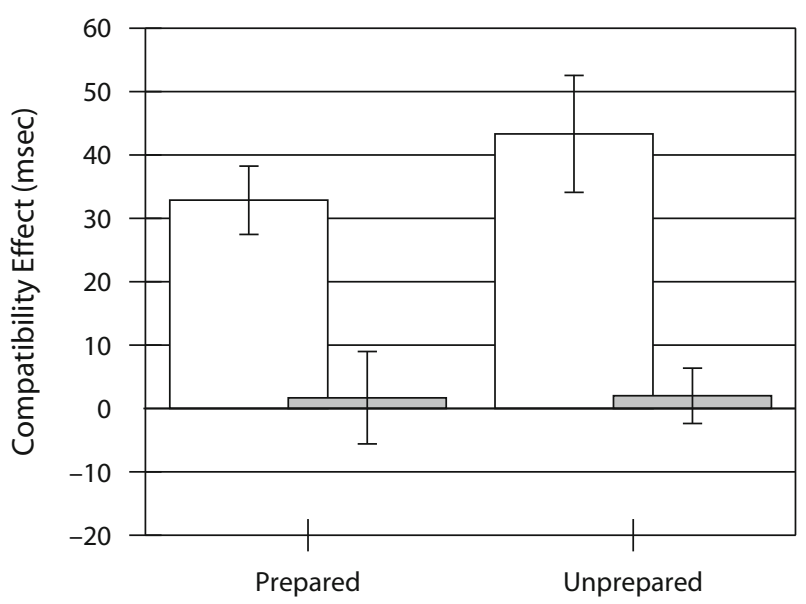

Foil Dimension

B

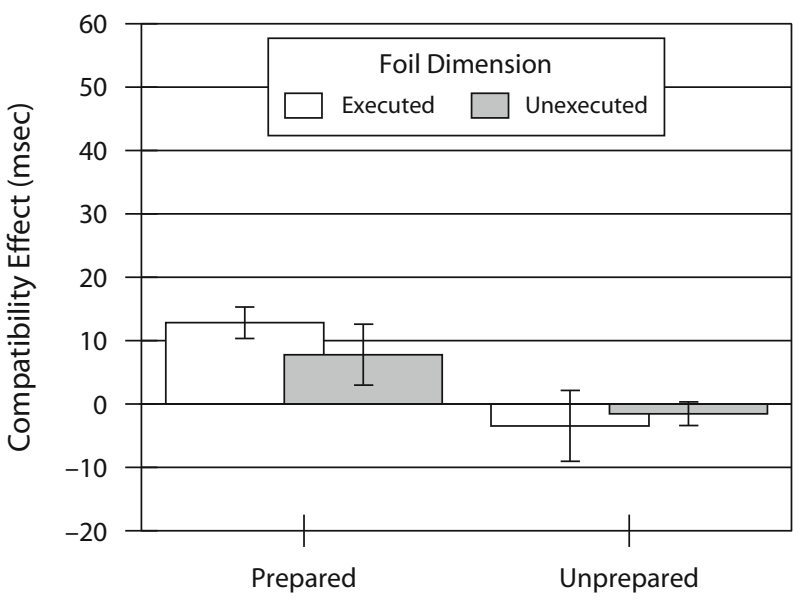

Foil Dimension

C

Target Cued

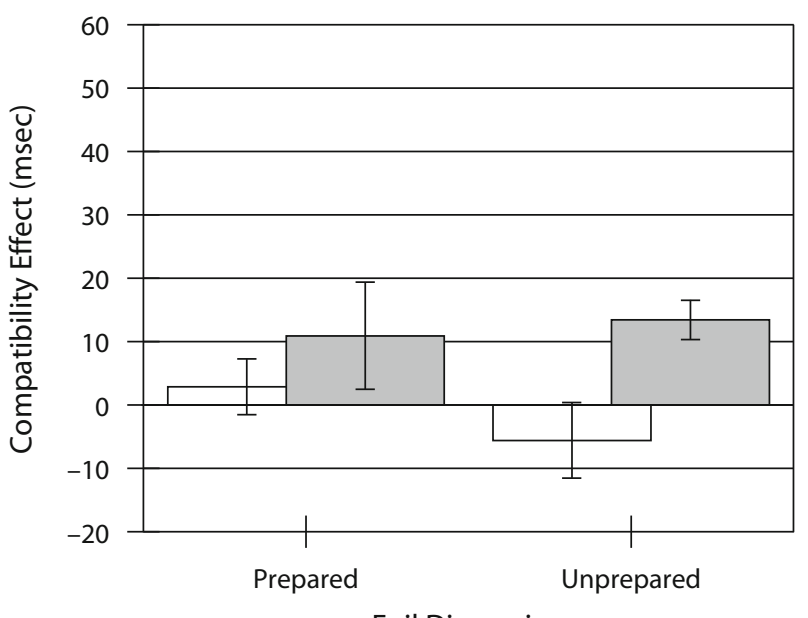

Foil Dimension

Figure 3. Compatibility effects in Experiment 2. Error bars indicate one standard error. was cued $(3.6 \%$ errors $)[F(2,90)=7.61, p<.001]$ and were more accurate when the target was of the prepared for task type $(2.5 \%$ errors $)$ than when it was of the unprepared for task type $(3.5 \%$ errors) $[F(1,45)=9.08, p<$ $.01]$. However, only cue location affected the magnitude of compatibility effects on error rates $[F(2,90)=9.23$, $p<.001]$ : The participants made $2.1 \%$ more errors for congruent than for noncongruent foils when the foil was cued, but this difference was only $0.2 \%$ when a neutral item was cued and only $0.1 \%$ when the target was cued. Compatibility effects were not significantly influenced by preparation, execution, their two-way interaction, or their interactions with cue location $\left(F_{\mathrm{S}}<1\right)$.

\section{Discussion}

Experiment 1 indicated that, in the absence of selective advance preparation for one dimension (i.e., one task) or the other, compatibility effects were much larger for foil dimensions relevant to the task being executed. The aim of Experiment 2 was to determine whether a similar pattern would hold when participants systematically prepared for a particular dimension. It is important to note, first, that both our attentional cuing manipulation and our task preparation manipulation had large main effects. Thus, our cuing manipulation appears to have effectively drawn attention to the location of the cue. Even more important for the purpose of this experiment, the participants appear to have prepared for the higher probability task type.

Although differences in preparation occurred, they had little impact on the overall pattern of compatibility effects. For cued (i.e., attended) foils, the executed dimension produced large compatibility effects even when it was not prepared for; conversely, the unexecuted dimension produced no compatibility effect even when it was prepared for.

\section{EXPERIMENT 3}

In Experiment 2, the target sometimes appeared at the cued location ( $25 \%$ of the trials). It is possible, therefore, that the participants not only reflexively attended that location, but sometimes also selected that object as a target, even when it was not in fact a target. It is even possible that this commitment to the cued location is the only reason that we observed compatibility effects from the executed but unprepared for dimension of a cued foil (since, according to Remington \& Folk, 2001, when an object is selected, both dimensions produce compatibility effects). In Experiment 3, therefore, we replicated Experiment 2, but in a design in which the target never appeared at the cued location. Accordingly, the participants had even less incentive to ever select that object and submit it to response mechanisms.

\section{Method}

Participants. Thirty-eight students from local colleges participated for pay or course credit. The first set of four blocks was biased toward the identity task for half of the participants (19) and toward the orientation task for the other half (19). No participants made more than our cutoff criterion of $10 \%$ errors or had a mean RT more than three $S D$ s above the mean for all the participants. 
Stimuli, Procedure, and Analysis. The stimuli, procedure, and analysis were identical to those in Experiment 2, except for the following: (1) All the cues appeared at the foil location (thus, the factor of cue location was removed from the analysis), and (2) to increase the efficiency of data collection, trial sequences were constrained so that there were never two trials of the unprepared for task type in a row.

\section{Results}

Our attempt to manipulate preparation via blocking of task bias was again successful. Mean RT was $578 \mathrm{msec}$ when the target was of the type the participants prepared for but $657 \mathrm{msec}$ when it was of the unprepared for type $[F(1,37)=171.96, p<.001]$.

The compatibility effect for each combination of preparation and execution was calculated as in Experiment 2. The resulting compatibility effects are shown in Figure 4. We conducted an ANOVA to assess the influence of preparation and execution on compatibility effects. As in Experiments 1 and 2, there was a significant main effect of execution $[F(1,37)=13.64, p<.001]$. Again, there was no effect of preparation $(F<1)$. However, unlike in Experiment 2, preparation did interact with execution $[F(1,37)=18.89$, $p<.001]$; substantial compatibility effects were seen only for foil dimensions that were both prepared for and executed. Again, we were primarily interested in the preparedfor-unexecuted and unprepared-for-executed conditions. Planned $t$ tests were performed to assess these effects. The 5-msec compatibility effect in the prepared-for-unexecuted case was not significant $[t(37)=1.20]$. However, the 12msec compatibility effect in the unprepared-for-executed condition was significant $[t(37)=2.30, p<.05$ ]. [Unlike in Experiment 2, these two effects were not significantly different from each other; $t(37)<1$.]

The overall error rate was again quite low (4.0\%). Echoing the RT results, the participants made $3.1 \%$ errors when the task was prepared for but $5.0 \%$ when it was not prepared for $[F(1,37)=19.64, p<.001]$. Neither preparation $[F(1,37)=1.36]$ nor execution $(F<1)$ had a significant

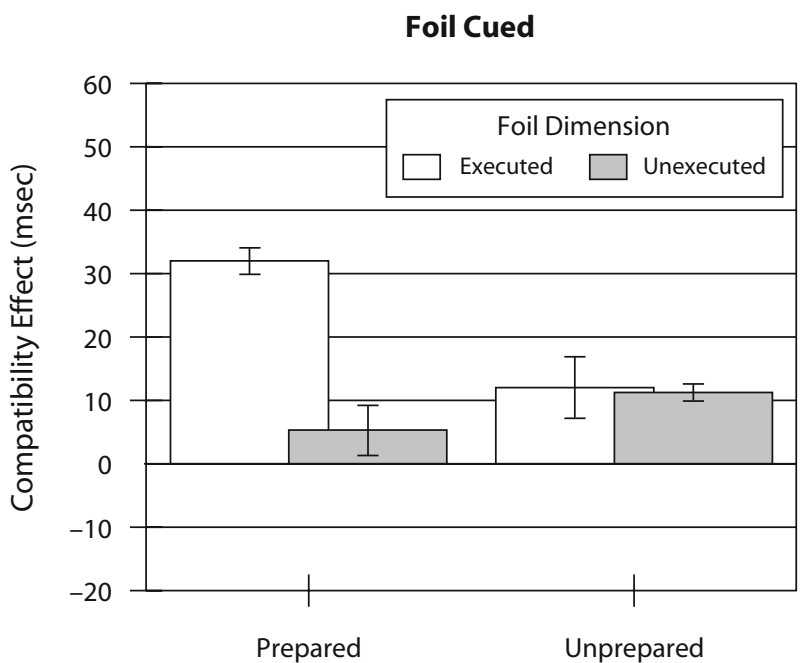

Foil Dimension

Figure 4. Compatibility effects in Experiment 3. Error bars indicate one standard error. impact on the congruence effect for errors. However, their interaction approached significance $[F(1,37)=3.39, .05<$ $p<.10$ ]. Interestingly, congruence of the prepared for but unexecuted dimension had the largest effect $(2.5 \%)$, whereas an unprepared-for-unexecuted dimension had little effect $(0.9 \%)$, with prepared-for-executed (1.4\%) and unpreparedfor-executed $(1.7 \%)$ falling somewhere in between.

\section{Discussion}

Once again, we obtained a large effect of preparation on raw RTs, indicating that the participants did prepare in advance for the more likely task. And once again, we obtained strong compatibility effects on RT from the executed dimension of the foil, stronger than those from the unexecuted dimension. Furthermore, these effects were statistically significant even when participants prepared to perform the unexecuted task.

One new finding, however, was that we now also obtained an interaction between the effects of preparation and execution. Stronger compatibility effects were seen when a foil dimension was both prepared for and executed than when it was executed but not prepared for. Although this suggests a role for preparation in producing these cross-dimensional compatibility effects, it is still the case that (1) significant compatibility effects were generated by the executed dimension of the foil, regardless of preparation, and (2) preparation once again was not sufficient to produce compatibility effects from an unexecuted foil dimension.

\section{EXPERIMENT 4}

In the previous experiments, the compatibility effect from the executed foil dimension was robust. In contrast, the compatibility effect from the unexecuted foil dimension was generally quite small. It is important to note, however, that when an executed foil dimension was compatible, it always had the same feature as the target on the executed dimension (while differing on the unexecuted dimension). For instance, when the participants responded to the identity of an upright $T$, a foil that was compatible on the executed dimension was also a $T$. When the unexecuted dimension was compatible, however, that feature could never exactly match the relevant target feature. For instance, when the participants responded to the identity of an upright $T$, the unexecuted foil feature was a left or a right slant.

It seems unlikely that the lack of a compatibility effect from the unexecuted dimension could have been due to the fact that the features were not identical, given that there are many previous examples of strong compatibility effects without physical similarity (Eriksen \& Eriksen, 1979; see Cohen \& Shoup, 1997, for a discussion). A notable example is Remington and Folk (2001), in which the unexecuted feature of the target produced very large compatibility effects without physical identity. Nevertheless, it is possible that the compatibility effect from the executed foil dimension would have been absent if not for the physical identity of the corresponding features. In other words, the degree of processing of the executed foil dimension might be just as shallow as that for the unexecuted foil dimension, but even that shallow level might be strong 
enough to produce a compatibility effect on a physically identical target feature.

To address this possibility, in the present experiment, physical similarity between the target and the relevant foil dimension was removed. If foil processing is inherently shallow, the lack of physical identity should eliminate compatibility effects from the executed foil dimension.

\section{Method}

Participants. Forty-three students from local colleges participated for pay or course credit. Of these, 1 was excluded from analysis for making more than $10 \%$ errors.

Stimuli and Procedure. Except as noted, the stimuli and procedure were the same as those in Experiment 3. As in the previous experiments, the participants performed one of two tasks: a letter task or an orientation task. However, in this experiment, the letter task required distinguishing vowels $(A$ and $E$ ) from consonants $(N$ and $T)$. The participants pressed the "." key for vowels and the " " key for consonants. In the orientation task, the participants determined whether the orientation of an elongated rectangle was slanted $\left(45^{\circ}\right.$ to the right or left) or not slanted (horizontal or vertical; see Figure 5). The participants pressed the "." key for slanted rectangles and the "/" key for nonslanted rectangles.

As in the previous experiments, two distractors and a foil were presented simultaneously with the target. However, in this experiment, the two distractors were an $x$ and $\mathrm{a}+$, and the foil was drawn from the same set of characters as the target: the upright letters $A, E, N$, and $T$ and elongated rectangles (see Figure 5). Notice that this design represents a break from the previous experiments, in which each foil stimulus afforded both tasks (i.e., had features on both the executed and the unexecuted dimensions). In this experiment, any particular foil could instantiate a feature from the executed or unexecuted dimension, but not both. The reason for this constraint is that the letters might be somewhat difficult to identify when misoriented (e.g., an $N$ rotated $90^{\circ}$ looks like a $Z$ ). Likewise, the slant of a letter might be less readily apparent than the slant of a line. As in Experiment 3, the foil always appeared in the cued location.

Trials were grouped into 12 blocks of 66 trials. These blocks alternated between letter blocks and orientation blocks ( 21 participants began with a letter block and 21 with an orientation block). The first block of each type was practice. Each block consisted of a mixture of 50 trials of one task and 16 trials of the other task. The participants were informed at the beginning of each block which type of task would occur most often. We will refer to the predominant task in a block as the prepared for task and the other task as the unprepared for task. The participants never saw 2 unprepared for task trials in a row. The first 2 trials of each block were always prepared for and were considered practice. Thus, there were 640 experimental trials divided into 10 blocks.

Across the first eight nonpractice blocks, each combination of task (orientation or letter), target $(A, E, N, T$, and each of the rect-

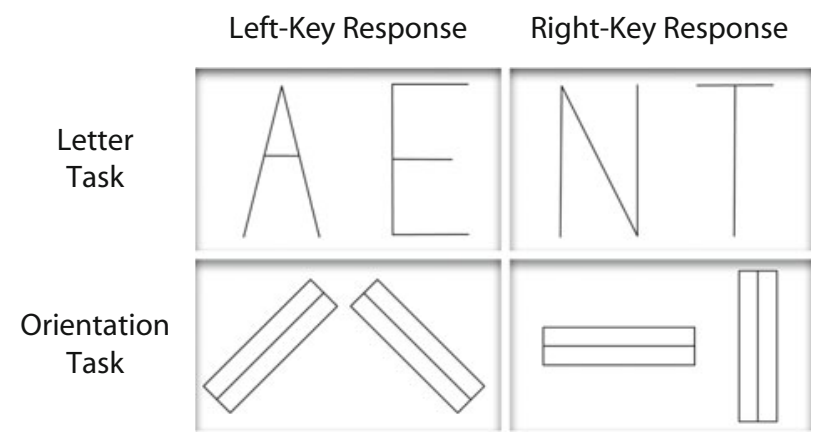

Figure 5. Stimuli used in Experiment 4.

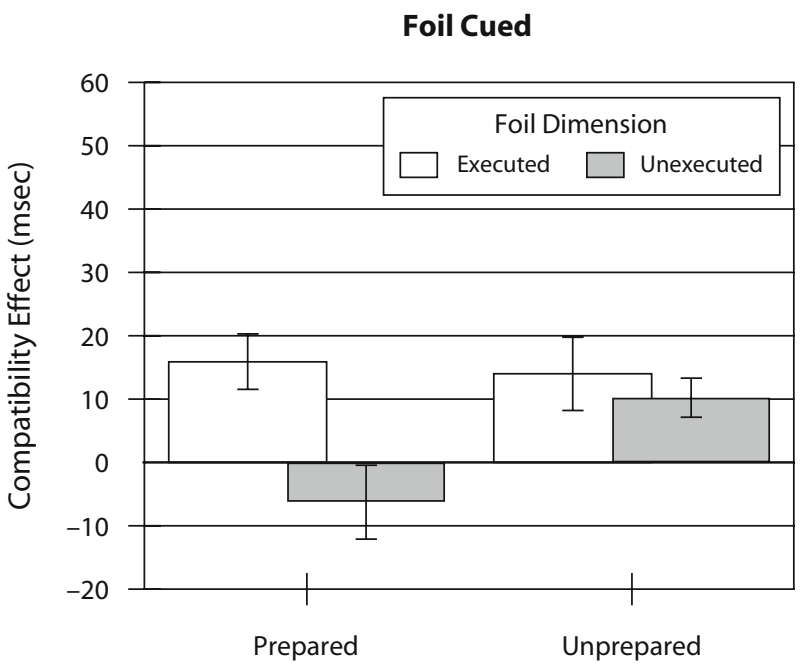

Foil Dimension

Figure 6. Compatibility effects in Experiment 4. Error bars indicate one standard error.

angles), and distractor ( $A, E, N, T$, and each of the rectangles) was presented six times for the prepared for condition and two times for the unprepared for condition. In the final two blocks, trials were selected randomly (subject to the constraint that there be 50 prepared for trials, including the 2 practice trials and 16 unprepared for trials), except that any unprepared for trials on which the participant had made an error earlier in the experiment were repeated.

\section{Results}

Data from conditions in which the foil was physically identical to the target were discarded. Our manipulation of preparation via blocking of task was once again successful. Mean RT was $652 \mathrm{msec}$ when the target was of the prepared for type but $689 \mathrm{msec}$ when it was unprepared for $[F(1,41)=90.66, p<.001]$.

The compatibility effect for each combination of preparation and execution was calculated as in the previous experiments. The resulting compatibility effects are shown in Figure 6. An ANOVA was conducted to assess the influence of preparation and execution on compatibility effects. As in Experiments 1,2, and 3, there was a significant main effect of execution $[F(1,41)=5.30, p<.05]$. Again, the effect of preparation was nonsignificant, although in this experiment, it approached significance $[F(1,41)=3.91$, $.05<p<.10]$. The interaction of preparation with execution was nonsignificant $[F(1,41)=2.58]$. Again, we were primarily interested in the prepared-for-unexecuted and unprepared-for-executed conditions. Planned $t$ tests were performed to assess these effects. The -6-msec compatibility effect in the prepared-for-unexecuted case was not significant $[t(41)<1]$. However, the 14-msec compatibility effect in the unprepared-for-executed condition was significant $[t(41)=2.30, p<.05]$. The difference between these two effects was significant $[t(41)=2.40, p<.05]$.

The overall error rate was again quite low $(3.3 \%)$. Echoing the RT results, the participants made $3.0 \%$ errors when the task was prepared for but $3.8 \%$ when it was unprepared for $[F(1,41)=7.69, p<.01]$. Preparation and 
execution both affected compatibility effects. Prepared for foils showed a normal $0.8 \%$ compatibility effect; however, unprepared for foils showed a $-0.6 \%$ compatibility effect $[F(1,41)=13.07, p<.001]$. Similarly, executed foils had the expected $0.7 \%$ compatibility effect, but unexecuted foils had a $-0.5 \%$ compatibility effect $[F(1,41)=4.73$, $p<.05]$.

\section{Discussion}

As in Experiments 1-3, compatibility effects from foil dimensions were influenced by whether the dimension was actually executed, but not by whether that dimension had been prepared for. The key difference is that, in this experiment, we found an effect of execution even when compatible foil features were no more similar (physically) to the target than were incompatible foil features. Thus, the effect of the executed dimension of the foil is robust and not based on only a shallow processing of the foil.

\section{GENERAL DISCUSSION}

To better understand visual attention, numerous studies have examined the effects of foils (defined as irrelevant stimuli with properties associated with potential responses) on RTs to target stimuli (e.g., Driver \& Baylis, 1989; Eriksen \& Hoffman, 1973; Flowers \& Wilcox, 1982; Grice \& Gwynne, 1985; Kramer \& Jacobson, 1991; Miller, 1991; Shaffer \& LaBerge, 1979). These studies have almost universally shown shorter RTs and lower error rates when the foils were compatible, rather than incompatible, with the target. However, these studies have focused on how the foils are processed, without regard to specific stimulus dimensions. Three more recent studies examined compatibility effects as a function of foil dimension. They showed that compatibility effects do not occur for foil dimensions that are irrelevant to the judgment made on the target (Cohen \& Shoup, 1997; Maruff et al., 1999), even when attention is deliberately drawn to the foil using an exogenous cue (Remington \& Folk, 2001). The inability of the irrelevant dimension of an attended foil to produce a compatibility effect is surprising, given that irrelevant dimensions of target objects can produce very large compatibility effects (Remington \& Folk, 2001). The four experiments reported here investigated the cause of this phenomenon, by disentangling the role of preparation from the role of execution.

One might have expected that a particular stimulus dimension would produce much larger compatibility effects when participants prepared to respond to it than when they did not. For instance, preparation should preactivate the pathway from the prepared for dimensions to the associated responses. The present experiments, however, did not confirm this expectation. Although the participants in Experiments 2-4 prepared to respond to one dimension of the target (as confirmed by shorter RTs to targets with this dimension), this preparation produced little in the way of compatibility effects from that dimension of the foil. Instead, in all four experiments, compatibility effects were produced primarily by the dimension on which the final target judgment was actually executed.
This result is even more surprising when you consider that attention is directed to the foil before it is directed to the target. To be concrete, consider a trial on which a participant prepares in advance to respond to the orientation dimension but the target later turns out to be an upright $T$ (i.e., requiring a response based on letter identity). Further suppose that the cue happens to draw attention to the foil stimulus. In such a condition, attention is presumably focused initially on the foil when the foil and target appear. The foil therefore should initially be processed according to the participant's initial task set (i.e., orientation). Attention then shifts to the target, and only at this point does the participant become aware that he or she must perform the letter (rather than orientation) judgment. By the time the participant has this realization, the foil is presumably no longer attended. Nevertheless, it is the letter dimension of the foil, not its orientation, that affects the target response. It appears, therefore, that letter identity was extracted from the foil even while the participant was still under the impression that the task would be orientation. Thus, as Duncan (1984) proposed, it appears that all the features of an object can be identified simultaneously. Interestingly, this appears to happen even when the observer believes the feature not to be task relevant.

\section{Implications}

The data presented here indicate that task execution, rather than task preparation, governs which dimension of a foil stimulus produces compatibility effects. This finding places important constraints on theories of attentional selection. In particular, to explain the finding that prepared for dimensions do not necessarily produce large compatibility effects, it must be assumed that features do not directly activate their associated responses (or do so very weakly). Instead, it appears that features activate responses primarily after an object has been selected as the target. Although the relevant features of unselected objects (e.g., foils) do not directly activate responses, they can indirectly produce compatibility effects by influencing the processing of the corresponding feature of the selected target object. For example, an attended foil slanted to the right can impede identification of the target as being slanted to the left. Below, we will make use of these constraints to develop a model of compatibility effects.

\section{Toward an Integrative Model of Compatibility Effects}

As was noted in the introduction, many previous studies have examined the influence of irrelevant stimuli or irrelevant features on target responses. On the surface, some of these results appear to contradict one another. In this section, we will demonstrate that these effects are, in fact, generally consistent. We will do so by presenting a model of how response decisions are made in the presence of multiple objects with multiple features. The model is not specified at a level of detail that allows for precise numerical predictions. However, it does provide an explanatory framework that illustrates how the consistency effects could arise from interactions between separate, distinct selection operations. 
The model. A generic representation of our spaceobject-task selection (SOTS) model is shown in Figure 7A. It consists of two levels of representation: semantic codes and response mappings. The connections made at the semantic code level are those that the participant brings to the experiment (e.g., those that allow the identification of letters and colors, and those between highly associated concepts). The connections made at the response mapping level are those specific to the assigned task (e.g., associating a particular letter with a particular buttonpress). An object can have several labels (e.g., RED, $T$, and vertical), but usually only one label per dimension (e.g., either $T$ or $L$, but not both). As a result, values on any single dimension inhibit one another, whereas values on different dimensions generally do not. Object files consist of a bound collection of features from different dimensions, represented at this semantic level. The representation of the response mapping indicates the task to be performed, allowing semantic codes assigned to stimuli to be mapped onto the semantic codes of potential responses.

In the SOTS model, the flow of information is orchestrated by three distinct selective operations. Spatial
A Generic Form of the Model

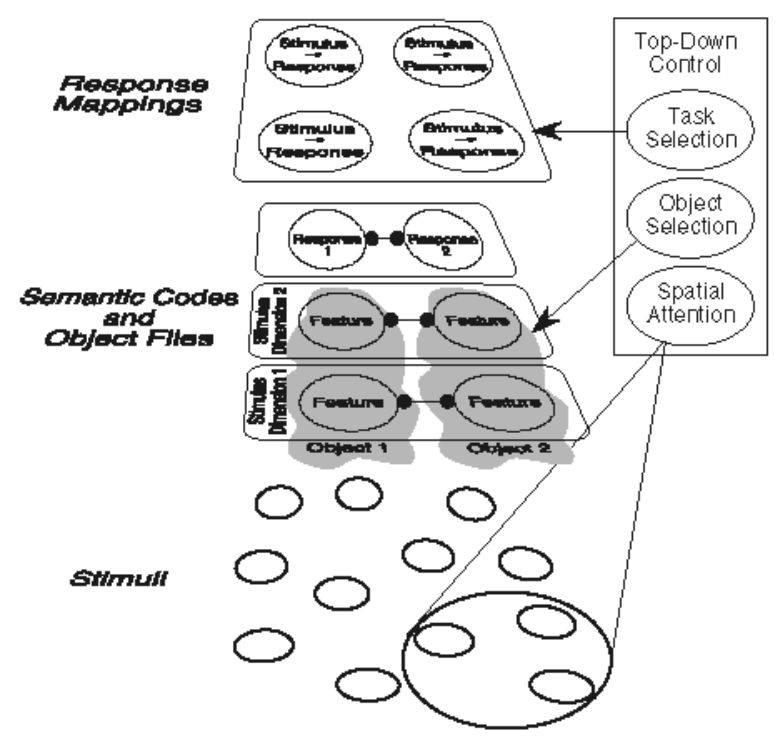

C

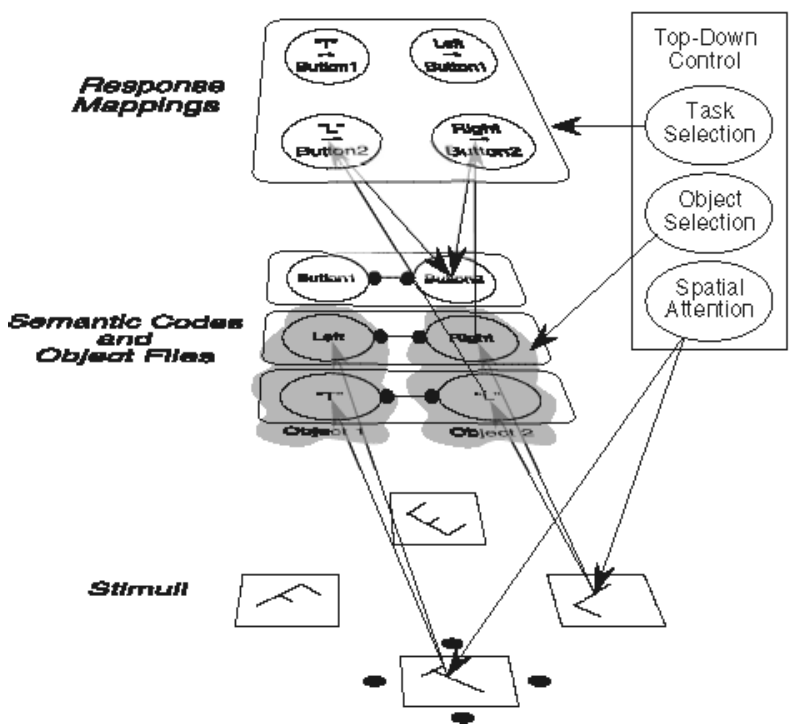

B

Experiment 4

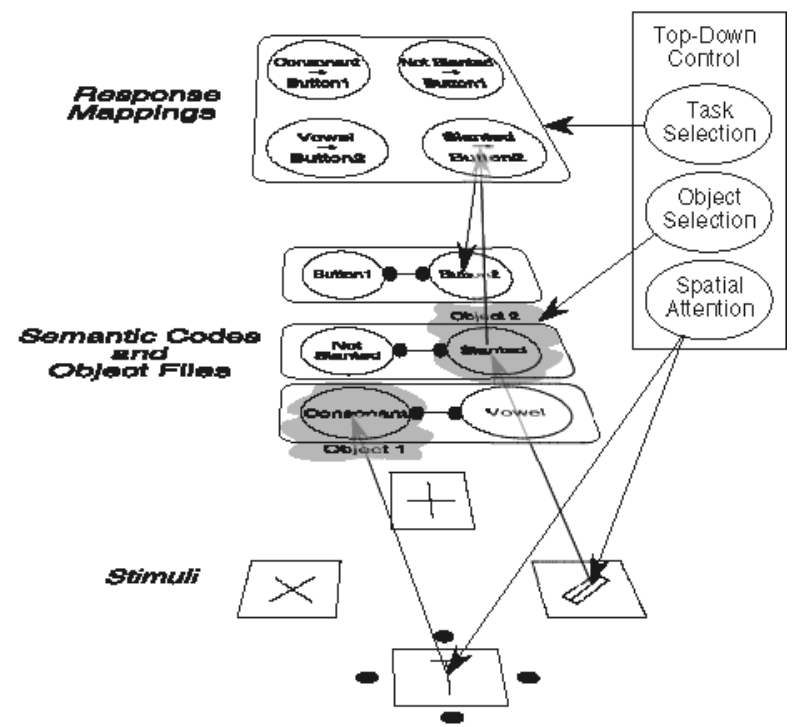

D

\section{Separated Stroop}

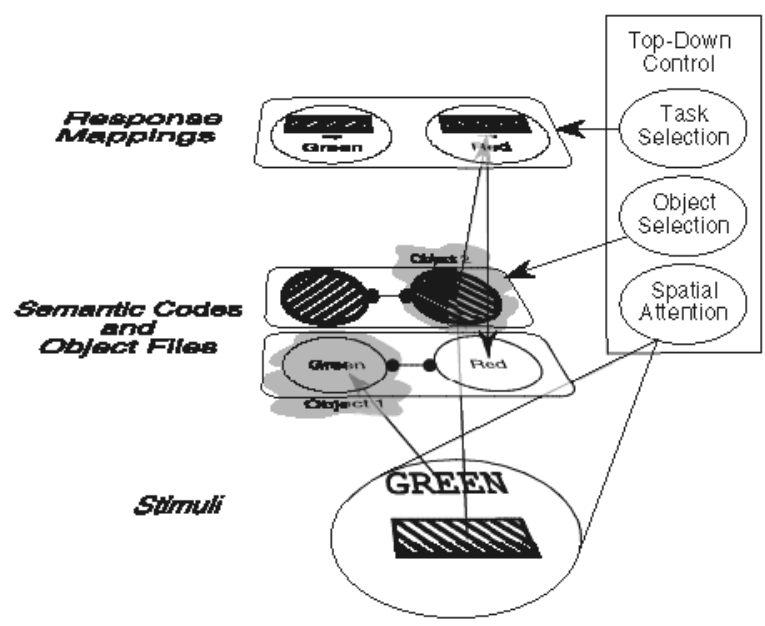

Figure 7. Schematic of the proposed model. (A) Generic form of the model. (B) Information flow in the model, given the task used in Experiment 4. (C) Information flow in the model, given the task used in Remington and Folk (2001). (D) Information flow in the model, given a Stroop task with color separated from words. 
attention governs which object(s) are assigned semantic codes. Our data argue that multiple features can be assigned semantic codes simultaneously; we have no data on what the upper limit might be on such processing. The model currently assumes that all features are assigned semantic codes. Object selection governs which object's semantic codes are mapped to response codes. Task selection determines which response mappings are applied to this selected object. These three selective operations are assumed to strongly modulate the flow of information; however, it is not necessary to assume that nonselected representations are completely suppressed.

Relation to other models. The SOTS model shares properties with the dimensional-action (DA) model (Cohen \& Magen, 1999; Cohen \& Shoup, 2000; Feintuch \& Cohen, 2002) and the translation model developed to explain the Stroop effect (Durgin, 2003; Virzi \& Egeth, 1985). In particular, SOTS follows the DA model (which in turn follows the feature integration model of Treisman \& Gelade, 1980) in assuming that intradimensional competition occurs before object-based selection. The DA model, however, does not have any obvious mechanism by which interdimensional compatibility effects, such as the Stroop effect, can occur. The object selection method proposed by Feintuch and Cohen (2002; see also Cohen \& Shoup, 2000) does generate Stroop effects when both dimensions are integrated into the same object but does not explain the numerous reports of Stroop effects when the word is separated from the color patch (Brown et al., 2002; Brown, Roos-Gilbert, \& Carr, 1995; Dyer, 1973; Gatti \& Egeth, 1978; Kahneman \& Chajczyk, 1983; Lachter et al., 2008; MacLeod, 1991; Merikle \& Gorewich, 1979). Their method also does not explain why the word interferes with color naming but the color does not interfere with word naming (Durgin, 2000, 2003; MacLeod, 1991; Stroop, 1935).

To account for these effects, the SOTS model incorporates an assumption, borrowed from translation theory (Durgin, 2003; Virzi \& Egeth, 1985), that the semantic representation of a stimulus can be the same as the semantic representation of a response. Words are the prototypical example of such stimuli; in the SOTS model, the semantic code of a written word used in reading is taken to be the same as the semantic code of the spoken word used in formulating a speech plan. As we will see, this direct connection of stimulus to response through a single semantic code allows the model to capture results from many different variations on the Stroop effect. Our model also differs from the DA model in that it allows for several types of selection occurring at different levels (see also Johnston et al., 1995).

Our model resembles Bundesen's (1987) theory of visual attention (TVA) model and Logan and Gordon's (2001) executive control of TVA model, both of which allow for attentional selection at multiple levels. These models, however, do not distinguish between spatial attention and object selection, a distinction that seems necessary in order to explain the tripartite distinction between spatially unattended foils (which produce little or no compatibility effect), spatially attended foils (which produce compatibility effects primarily from the executed dimension, not the unexecuted dimension), and targets (which produce strong compatibility effects from all dimensions; see Remington \& Folk, 2001).

The SOTS model is consistent with the present results. Figure 7B illustrates how stimuli such as those used in Experiment 4 are processed, according to the SOTS model. We presume that attention is initially drawn to the cue and then moves to the target after it appears. Thus, only the distractor in the cued location and the target receive spatial attention, and only their features are assigned semantic codes. The model thus explains the finding that compatibility effects from the foil occur primarily when it is cued (Experiments 1 and 2; see also Remington \& Folk, 2001). Compatibility effects from the relevant dimension of the foil are generated by the inhibitory links between semantic codes within the same dimension. Because there are no such inhibitory links between dimensions, the unexecuted dimension of the foil does not affect processing of the executed dimension of the target at the semantic code level. The nontarget objects are not selected for further processing, so neither dimension of the foil is mapped to a response. Thus, the SOTS model can explain the fact that compatibility effects from nontarget objects occur primarily for the executed dimension (Experiments 1-4; Cohen \& Shoup, 1997; Maruff et al., 1999; Remington \& Folk, 2001).

The SOTS model is consistent with facilitation by items that are not physically similar. It has long been known that stimuli assigned to the same response will often facilitate each other, even without any physical similarity (Eriksen \& Eriksen, 1979; see Experiment 4 above). In the present Experiment 4, for example, the participants were shown four possible oriented rectangles and four possible letters. However, they were encouraged (both through the instructions and through the requirements of the task) to encode the rectangles as slanted or nonslanted and the letters as consonants or vowels. Compatibility effects were observed between the various orientation stimuli and between the various letter stimuli, despite the fact that stimuli belonging to the same category were not more physically similar than those belonging to different categories. In the model, compatibility effects between nonidentical objects or features arise because of competition at the level of semantic codes (see Figure 7B), and thus it is the similarity of the semantic codes assigned by the participants in the context of the experiment, rather than physical similarity, that determines whether compatibility effects will be generated.

It should be noted that effects such as those seen in Experiment 4 are more typically explained by invoking competition between responses. However, compatibility effects are dependent on responses' being associated with higher level semantic categories (as was the case in Experiment 4), suggesting that it is competition between these categories, and not the responses, that is primarily responsible for the compatibility effects. Shaffer and LaBerge (1979), for instance, asked participants to press one button if a centrally presented word belonged 
to one of two categories (e.g., clothing or trees) and a second button if it belonged to one of the other two categories (e.g., furniture or metals). The key measure was the size of the compatibility effects from flanking foil words. When the two categories assigned to the same response did not have a plausible superordinate (e.g., clothing and trees), Shaffer and LaBerge found that flankers from the same category as the target facilitated responses, but flankers from the other category assigned to that response did not. In contrast, when the two categories assigned to the same response had a plausible superordinate (e.g., clothing and furniture, both of which are man-made artifacts), facilitation occurred between all items assigned to the same response. There was little added benefit for flankers from the same nominal category as the target (see also Campbell \& Proctor, 1993). This finding suggests that Shaffer and LaBerge's participants chose their own categorization of the stimuli and that facilitation/inhibition occurred between these semantic codes. Although such effects fall naturally out of the proposed model, they are difficult to explain if compatibility effects are generated primarily at the response level. Note that in Experiment 4, each response grouping had a plausible superordinate category (consonants vs. vowels; slanted vs. perpendicular). If we had used categories without such superordinates, we might have obtained different results (i.e., no compatibility effects from either dimension of the foil).

The SOTS model is consistent with the contingency of cross-dimensional effects on object selection. Although the irrelevant dimension of a foil object has little effect on responses, the irrelevant dimension of a target object can have very large effects (Remington \& Folk, 2001). This finding echoes the earlier finding (using a very different paradigm) of Duncan (1984) that people can report two features of the same object more easily than two features of two different objects. In our model, this dichotomy arises from the fact that all features of a selected object are passed to the response mappings (see Figure 7C). If there are active mappings for more than one feature of the target, each feature will activate a separate response mapping, which will, in turn, activate the semantic code of its associated response. If the tasks also use the same response set (as in the present experiments), compatibility effects will arise. Note that nonselected objects do not affect responses in this way, because they are not passed to the response-mapping level.

The SOTS model is consistent with the results from the "separated Stroop" paradigm. Stroop effects are of special interest here because they appear to challenge the generality of the present findings. In particular, when the word and the color are separated into two objects (e.g., participants name the color of a rectangle, and a black word is printed above or below it), the word still affects responses to the color (Brown et al., 2002; Brown et al., 1995; Dyer, 1973; Gatti \& Egeth, 1978; Kahneman \& Chajczyk, 1983; Lachter et al., 2008; Merikle \& Gorewich, 1979). Thus, an irrelevant dimension of an irrelevant object (i.e., the word's identity) that is both unprepared for and unexecuted can sometimes affect responses, seemingly contrary to the present findings, as well as those of Cohen and Shoup (1997), Maruff et al. (1999), and Remington and Folk (2001). However, we believe that these separated Stroop effects are a special case and that they can be gracefully captured by the SOTS model (see Figure 7D).

Note that all attended objects (and possibly, some unattended ones ${ }^{3}$ ) activate semantic codes (i.e., are identified), even if they are not ultimately selected for response. According to the SOTS model, therefore, even a spatially separated color word can activate a semantic word code (in Figure 7D, the word GREEN activates the semantic code for the word green). Assuming that the semantic code for the stimulus GREEN is the same as (or at least closely associated with) the semantic code for the vocal response "green," compatibility effects will occur. This mechanism has been proposed independently by other authors to explain certain variations of the Stroop effect (Durgin, 2000, 2003; Virzi \& Egeth, 1985). In our example, when participants are asked to respond to a red rectangle by saying the word "red," the semantic code for the color red must be translated into the semantic code for the word red (via the response mappings). The semantic code from the word red is inhibited by the semantic code for the word green, giving rise to a Stroop effect. Thus, our model can explain why Stroop effects occur even when the word does not belong to the target object.

Two findings deserve mention because they appear to contradict the SOTS model. First, Melara and Mounts (1993) demonstrated that slowing color identification (e.g., by using smaller print) reduces the standard Stroop effect yet increases the reverse Stroop effect (colors influencing word identification). Melara and Mounts claimed that this finding is contrary to the translation theory, on which our model is partially based. However, the observed reductions in the standard Stroop effect were highly dependent on response modality: A much larger reduction in font size was necessary to reduce Stroop effects with verbal responses than with manual responses. This finding suggests that Stroop effects are dependent on the degree to which a given stimulus activates a particular response. This is precisely what causes Stroop effects in our model's implementation of translation theory. Weakening the activation of the stimulus (as Melara \& Mounts, 1993, did) has the same effect as weakening the association between stimulus and response. Thus, our model captures these effects in much the same way as it explains other variations of the Stroop effect.

A second finding that appears to contradict the SOTS model is that of Mordkoff and Yantis (1993). Mordkoff and Yantis used a go/no-go paradigm in which participants were to respond only if the target contained either the color green or the shape $\mathrm{X}$. They found that when the target contained both the color green and the shape X, responses were faster than could be explained by simply assuming that the response was generated by the faster of these two dimensions. Mordkoff and Yantis reasoned that, for this to be true, both dimensions must simultaneously contribute to the activation of the response. 
Interestingly, from our perspective, this result held even when the color and shape were separated (the color was a frame around the shape in Experiment 2, and there was a separate color patch below fixation in Experiment 3). Thus, in the Mordkoff and Yantis experiments, two dimensions from separate objects can simultaneously activate a response (known as coactivation). This result seems to run counter to the SOTS model and the experiments discussed above.

Before concluding that the SOTS model cannot handle their results, however, it should be noted that the task used by Mordkoff and Yantis (1993) differed from those considered above in one crucial manner: All the stimuli were targets (there were no distractor or foil items). As a result, the participants did not need to inhibit responses to foil items. The obvious strategy for dealing with such a display is to select the entire display to be mapped onto a response. If the participants adopted this strategy, the SOTS model would predict that both target dimensions would influence the response on every trial just as they do when they nominally belong to the same object (see The SOTS Model Is Consistent With the Contingency of Cross-Dimensional Effects on Object Selection section above). In fact, Feintuch and Cohen (2002) conducted a series of experiments very similar to those conducted by Mordkoff and Yantis, varying the degree to which the two targets would be simultaneously selected by varying the degree to which they were perceived as one object. If, as was argued by Feintuch and Cohen, the participants in the Mordkoff and Yantis experiments selected both display items, either as a single object or as two separate objects, the SOTS model predicts that the associated response mappings would be activated in parallel, resulting in coactivation, as found by Mordkoff and Yantis.

\section{CONCLUSIONS}

Recently, several studies have shown that only certain dimensions of attended nontarget objects can generate compatibility effects. We have presented four experiments that show that the effective dimensions are not those that people prepare for but, rather, those to which they eventually respond. This dominance of execution over preparation suggests that encodings of stimulus features are not directly linked to their corresponding responses (at least, not very strongly). Instead, it appears that only selected objects directly influence response mechanisms. Furthermore, the fact that compatibility effects occur for executed dimensions, even when people do not expect them to be task relevant, suggests that all features of attended stimuli are initially identified. To explain our findings and those of previous studies, we have proposed a model in which three distinct filtering operations are used to map stimuli onto responses: spatial attention, object selection, and task selection.

\section{AUTHOR NOTE}

Research support was provided by the Airspace Operations Systems Project of NASA's Airspace Systems Program, a postdoctoral fellow- ship to J.L. from the National Research Council, and Air Force Office of Scientific Research Grant FA9550-07-1-0356 to R.W.R. We thank Frank Durgin and Rob McCann for their helpful discussions of the Stroop effect. Correspondence concerning this article should be addressed to J. Lachter, NASA Ames Research Center, MS 262-4, Moffett Field, CA 94035 (e-mail: joel.b.lachter@nasa.gov).

Note-Accepted by the previous editorial team, when Thomas H. Carr was Editor.

\section{REFERENCES}

Allport, D. A., Tipper, S. P., \& Chmiel, N. R. J. (1985). Perceptual integration and post-categorical filtering. In M. I. Posner \& O. S. M. Marin (Eds.), Attention and performance XI (pp. 107-133). Hillsdale, NJ: Erlbaum.

Besner, D., Risko, E. F., \& SkLair, N. (2005). Spatial attention as a necessary preliminary to early processes in reading. Canadian Journal of Experimental Psychology, 59, 99-108.

Besner, D., \& Stolz, J. A. (1999). What kind of attention modulates the Stroop effect? Psychonomic Bulletin \& Review, 6, 99-104.

Broadbent, D. E. (1958). Perception and communication. New York: Oxford University Press.

Brown, T. L., Gore, C. L., \& CARR, T. H. (2002). Visual attention and word recognition in Stroop color naming: Is word recognition "automatic"? Journal of Experimental Psychology: General, 131, 220-240.

Brown, T. L., Roos-GilberT, L., \& CARR, T. H. (1995). Automaticity and word perception: Evidence from Stroop and Stroop dilution effects. Journal of Experimental Psychology: Learning, Memory, \& Cognition, 21, 1395-1411.

Bundesen, C. (1987). Visual attention: Race models for selection from multielement displays. Psychological Research, 49, 113-121.

Campbell, K. C., \& Proctor, R. W. (1993). Repetition effects with categorizable stimulus and response sets. Journal of Experimental Psychology: Learning, Memory, \& Cognition, 19, 1345-1362.

Cohen, A., \& Magen, H. (1999). Intra- and cross-dimensional visual search for single-feature targets. Perception \& Psychophysics, 61, 291-307.

Cohen, A., \& Shoup, R. (1997). Perceptual dimensional constraints in response selection processes. Cognitive Psychology, 32, 128-181.

Cohen, A., \& Shoup, R. (2000). Response selection processes for conjunctive targets. Journal of Experimental Psychology: Human Perception \& Performance, 26, 391-411.

Dark, V. J., Johnston, W. A., Myles-Worsley, M., \& Farah, M. J. (1985). Levels of selection and capacity limits. Journal of Experimental Psychology: General, 114, 472-497.

Desimone, R., \& Duncan, J. (1995). Neural mechanisms of selective visual attention. Annual Review of Neuroscience, 18, 193-222.

Driver, J., \& BAYLIS, G. C. (1989). Movement and visual attention: The spotlight metaphor breaks down. Journal of Experimental Psychology: Human Perception \& Performance, 15, 448-456.

DuNCAN, J. (1984). Selective attention and the organization of visual information. Journal of Experimental Psychology: General, 113, 501517.

Durgin, F. H. (2000). The reverse Stroop effect. Psychonomic Bulletin \& Review, 7, 121-125.

DuRgin, F. H. (2003). Translation and competition among internal representations in a reverse Stroop effect. Perception \& Psychophysics, 65, 367-378.

DYER, F. N. (1973). Interference and facilitation for color naming with separate bilateral presentations of the word and color. Journal of Experimental Psychology, 99, 314-317.

ERIKSEN, C. W., \& ERIKSEN, B. A. (1979). Target redundancy in visual search: Do repetitions of the target within the display impair processing? Perception \& Psychophysics, 26, 195-205.

ERIKSEN, C. W., \& Hoffman, J. E. (1973). The extent of processing of noise elements during selective encoding from visual displays. Perception \& Psychophysics, 14, 155-160.

Fanini, A., Nobre, A. C., \& Chelazzi, L. (2006). Selecting and ignoring the component features of a visual object: A negative priming paradigm. Visual Cognition, 14, 584-618.

FeIntuch, U., \& CoHEN, A. (2002). Visual attention and coactivation 
of response decisions for features from different dimensions. Psychological Science, 13, 361-369.

FLOWERS, J. H., \& WiLCOX, N. (1982). The effect of flanking context on visual classification: The joint contribution of interactions at different processing levels. Perception \& Psychophysics, 32, 581-591.

Folk, C. L., Remington, R. W., \& Johnston, J. C. (1992). Involuntary covert orienting is contingent on attentional control settings. Journal of Experimental Psychology: Human Perception \& Performance, 18, 1030-1044.

Frings, C., \& Wentura, D. (2006). Negative priming is stronger for task-relevant dimensions: Evidence of flexibility in the selective ignoring of distractor information. Quarterly Journal of Experimental Psychology, 59, 683-693.

GARNER, W. R. (1974). The processing of information and structure. Potomac, MD: Erlbaum.

Gatti, S. V., \& EGETh, H. E. (1978). Failure of spatial selectivity in vision. Bulletin of the Psychonomic Society, 11, 181-184.

Grice, G. R., \& Gwynne, J. W. (1985). Temporal characteristics of noise conditions producing facilitation and interference. Perception \& Psychophysics, 37, 495-501.

JAMES, W. (1890). The principles of psychology (1st ed., Vol. I). New York: Holt.

Johnston, J. C., McCann, R. S., \& Remington, R. W. (1995). Chronometric evidence for two types of attention. Psychological Science, 6, 365-369

Kahneman, D., \& ChaJczyK, D. (1983). Tests of the automaticity of reading: Dilution of Stroop effects by color-irrelevant stimuli. Journal of Experimental Psychology: Human Perception \& Performance, 9, 497-509.

Kramer, A. F., \& Jacobson, A. (1991). Perceptual organization and focused attention: The role of objects and proximity in visual processing. Perception \& Psychophysics, 50, 267-284.

Lachter, J., Forster, K. I., \& RuthrufF, E. (2004). Forty-five years after Broadbent (1958): Still no identification without attention. Psychological Review, 111, 880-913.

Lachter, J., Ruthruff, E., Lien, M.-C., \& McCann, R. S. (2008). Is attention needed for word identification? Evidence from the Stroop paradigm. Psychonomic Bulletin \& Review, 15, 950-955.

Logan, G. D., \& Gordon, R. D. (2001). Executive control of visual attention in dual-task situations. Psychological Review, 108, 393-434.

MACLEOD, C. M. (1991). Half a century of research on the Stroop effect: An integrative review. Psychological Bulletin, 109, 163-203.

Maruff, P., Danckert, J., Camplin, G., \& Currie, J. (1999). Behavioral goals constrain the selection of visual information. Psychological Science, 10, 522-525.

McCann, R. S., Folk, C. L., \& Johnston, J. C. (1992). The role of spatial attention in visual word processing. Journal of Experimental Psychology: Human Perception \& Performance, 18, 1015-1029.

Melara, R. D., \& Mounts, J. R. W. (1993). Selective attention to Stroop dimensions: Effects of baseline discriminability, response mode, and practice. Memory \& Cognition, 21, 627-645.

MerikLe, P. M., \& Gorewich, N. J. (1979). Spatial selectivity in vision: Field size depends upon noise size. Bulletin of the Psychonomic Society, 14, 343-346.

Miller, J. (1991). The flanker compatibility effect as a function of visual angle, attentional focus, visual transients, and perceptual load: A search for boundary conditions. Perception \& Psychophysics, 49, 270-288.

MoRdKOFF, J. T. (1998). Between-dimension flanker effects: A clarification with encouraging implications. Psychonomic Bulletin \& Review, 5, 670-675.

MoRdKofF, J. T., \& Yantis, S. (1993). Dividing attention between color and shape: Evidence of coactivation. Perception \& Psychophysics, 53, 357-366.
Mounts, J. R. W., \& Melara, R. D. (1999). Attentional selection of objects or features: Evidence from a modified search task. Perception \& Psychophysics, 61, 322-341.

Park, J., \& Kanwisher, N. (1994). Negative priming for spatial locations: Identity mismatching, not distractor inhibition. Journal of Experimental Psychology: Human Perception \& Performance, 20, 613-623.

Posner, M. I. (1980). Orienting of attention. Quarterly Journal of Experimental Psychology, 32, 3-25.

Remington, R. W., \& FolK, C. L. (2001). A dissociation between attention and selection. Psychological Science, 12, 511-515.

Ruthruff, E., Remington, R. W., \& Johnston, J. C. (2001). Switching between simple cognitive tasks: The interaction between top-down and bottom-up factors. Journal of Experimental Psychology: Human Perception \& Performance, 27, 1404-1419.

Shaffer, W. O., \& LaBerge, D. (1979). Automatic semantic processing of unattended words. Journal of Verbal Learning \& Verbal Behavior, 18, 413-426.

Shalev, L., \& Algom, D. (2000). Stroop and Garner effects in and out of Posner's beam: Reconciling two conceptions of selective attention. Journal of Experimental Psychology: Human Perception \& Performance, 26, 997-1017.

Stroop, J. R. (1935). Studies of interference in serial verbal reactions. Journal of Experimental Psychology, 18, 643-662.

TIPPER, S. P. (2001). Does negative priming reflect inhibitory mechanisms? A review and integration of conflicting views. Quarterly Journal of Experimental Psychology, 54A, 321-343.

Tipper, S. P., Weaver, B., \& Houghton, G. (1994). Behavioural goals determine inhibitory mechanisms of selective attention. Quarterly Journal of Experimental Psychology, 47A, 809-840.

Treisman, A. M., \& Gelade, G. (1980). A feature-integration theory of attention. Cognitive Psychology, 12, 97-136.

VIRZI, R. A., \& EGETH, H. E. (1985). Toward a translational model of Stroop interference. Memory \& Cognition, 13, 304-319.

Vogel, E. K., Woodman, G. F., \& LUCK, S. J. (2005). Pushing around the locus of selection: Evidence for the flexible-selection hypothesis. Journal of Cognitive Neuroscience, 17, 1907-1922.

Yantis, S., \& Johnston, J. C. (1990). On the locus of visual selection: Evidence from focused attention tasks. Journal of Experimental Psychology: Human Perception \& Performance, 16, 135-149.

\section{NOTES}

1. In these paradigms, the participant is usually presented an object with two features (e.g., a word printed in a specific color) and asked to judge one of those features (requiring selection of that particular feature). There are variants in which the features are embedded in different objects (e.g., a word and a color patch). In these variants, it is usually assumed that spatial attention is responsible for selecting the appropriate information on which to base the response.

2. Compatibility effects are defined as the impact on performance of whether the foil and target are associated with the same response or conflicting responses.

3. It is important to note that the SOTS model does not make specific predictions with respect to the degree of effectiveness of spatial attention. Consider the separated Stroop task, which has been used by a number of authors to examine whether spatial attention is required for identification of words (Besner \& Stolz, 1999; Brown et al., 2002; Brown et al., 1995; Kahneman \& Chajczyk, 1983; Lachter et al., 2008). There is still some controversy about whether the words are identified in a separated Stroop task under conditions in which the word is truly unattended. This controversy is about the efficiency of spatial selection, on which the SOTS model is neutral. Instead, the model describes how distinct modes of attentional selection interact, with special emphasis on explaining the pattern of compatibility effects across a wide range of paradigms. 


\section{APPENDIX}

Our purpose in conducting the experiments presented in this article was to assess the role of preparation and execution in the production of compatibility effects. As a result, the analyses presented focus on the effects of preparation and execution on compatibility. For the purpose of comparing our results with other results in the literature, however, this Appendix will provide more detailed analyses that present raw RTs and error rates and break out the contributions of the letter and orientation tasks.

\section{Detailed Analysis of Experiment 1}

In Experiment 1, four factors were manipulated orthogonally: task (letter vs. orientation), cue location (foil, neutral, or target), compatibility of the letter identity of the foil with the target (compatible or incompatible), and compatibility of the orientation of the foil with the target (compatible or incompatible). Mean RTs and error rates for each cell in this design are presented in Table A1. ANOVAs were performed on both RTs and error rates. The results of this ANOVA appear in Table A2.

Table A1

Mean Response Times (RTs, in Milliseconds) and Error Rates (\%E) in Experiment 1

\begin{tabular}{|c|c|c|c|c|c|c|c|c|c|}
\hline \multirow[b]{3}{*}{ Task } & \multirow[b]{3}{*}{ Cue } & \multicolumn{8}{|c|}{ Compatibility by Dimension (Letter/Orientation) } \\
\hline & & \multicolumn{2}{|c|}{$\mathrm{Com} / \mathrm{Com}$} & \multicolumn{2}{|c|}{$\mathrm{Com} / \mathrm{Inc}$} & \multicolumn{2}{|c|}{$\mathrm{Inc} / \mathrm{Com}$} & \multicolumn{2}{|c|}{ Inc/Inc } \\
\hline & & RT & $\% \mathrm{E}$ & RT & $\% \mathrm{E}$ & RT & $\% \mathrm{E}$ & RT & $\% \mathrm{E}$ \\
\hline \multirow[t]{3}{*}{ Letter } & Target & 593 & 2.1 & 599 & 3.6 & 596 & 2.4 & 593 & 1.4 \\
\hline & Foil & 635 & 3.6 & 654 & 5.7 & 666 & 3.4 & 668 & 5.7 \\
\hline & Neutral & 630 & 3.6 & 635 & 3.2 & 635 & 4.5 & 633 & 3.5 \\
\hline \multirow[t]{3}{*}{ Orientation } & Target & 554 & 0.9 & 561 & 1.1 & 552 & 1.3 & 565 & 1.2 \\
\hline & Foil & 594 & 1.5 & 638 & 1.8 & 608 & 1.3 & 643 & 1.5 \\
\hline & Neutral & 608 & 1.0 & 617 & 1.4 & 596 & 1.8 & 616 & 1.5 \\
\hline
\end{tabular}

Note-Com, compatible; Inc, incompatible.

Table A2

ANOVA Results for Experiment 1

\begin{tabular}{|c|c|c|c|c|c|}
\hline \multirow[b]{2}{*}{ Source } & \multirow[b]{2}{*}{$d f$} & \multicolumn{2}{|c|}{ Response Times } & \multicolumn{2}{|c|}{ Errors } \\
\hline & & $F$ & $p$ & $F$ & $p$ \\
\hline Task & 1,37 & 7.11 & .011 & 29.17 & .000 \\
\hline Cue & 2,74 & 241.17 & .000 & 7.08 & .002 \\
\hline Task $\times$ cue & 2,74 & 5.23 & .008 & 5.45 & .006 \\
\hline Letter & 1,37 & 6.66 & .014 & 0.00 & .981 \\
\hline Task $\times$ letter & 1,37 & 2.89 & .097 & 0.32 & .577 \\
\hline Cue $\times$ letter & 2,74 & 12.39 & .000 & 0.89 & .417 \\
\hline Task $\times$ cue $\times$ letter & 2,74 & 2.97 & .058 & 1.13 & .328 \\
\hline Orientation & 1,37 & 46.44 & .000 & 1.60 & .214 \\
\hline Task $\times$ orientation & 1,37 & 22.72 & .000 & 1.47 & .234 \\
\hline Cue $\times$ orientation & 2,74 & 7.26 & .001 & 3.50 & .035 \\
\hline Task $\times$ cue $\times$ orientation & 2,74 & 3.86 & .026 & 4.16 & .019 \\
\hline Letter $\times$ orientation & 1,37 & 1.40 & .244 & 4.47 & .041 \\
\hline Task $\times$ letter $\times$ orientation & 1,37 & 5.16 & .029 & 0.57 & .454 \\
\hline Cue $\times$ letter $\times$ orientation & 2,74 & 2.10 & .129 & 0.83 & .441 \\
\hline Task $\times$ cue $\times$ letter $\times$ orientation & 2,74 & 0.28 & .757 & 1.02 & .367 \\
\hline
\end{tabular}

The effect of task was significant. The participants were faster $(596 \mathrm{msec})$ and more accurate $(1.4 \%$ errors $)$ on the orientation task than on the letter task $(628 \mathrm{msec} ; 3.6 \%$ errors). Cue location was also significant (as reported in the text), as was the task $\times$ cue location interaction (the effect of task was $35 \mathrm{msec}$ and $3.1 \%$ errors when the foil was cued, $24 \mathrm{msec}$ and $2.3 \%$ errors when a neutral item was cued, and $36 \mathrm{msec}$ and $1.2 \%$ errors when the target was cued).

The effect of compatibility with the letter identity of the foil was significant in RTs; however, this effect must be looked at in the context of its interactions with task and cue location. When the task was the letter task and the foil was cued, there was a 23 -msec effect of letter compatibility. When the task was the orientation task and the foil was cued, there was a 10-msec effect of letter compatibility. In all other conditions, the effect of letter compatibility was between 1 and $-6 \mathrm{msec}$. Thus, letter compatibility effects are seen only when the foil is cued and are much larger for the letter task. These differences are reflected in the significant interaction of cue location with letter compatibility and the marginally significant interactions of task and letter compatibility and of task, cue location, and letter compatibility. Error rates were unaffected by letter compatibility or any of these interactions. 


\section{APPENDIX (Continued)}

Compatibility with the orientation of the foil acted similarly to compatibility with letter identity. There was an overall effect of orientation compatibility on RT, which was modulated by both task and cue location. There was a 40-msec effect of orientation compatibility in the orientation task when the foil was cued. This fell to $15 \mathrm{msec}$ when a neutral item was cued and $10 \mathrm{msec}$ when the target was cued. There was a 10-msec effect of orientation compatibility in the letter task when the foil was cued, but only a 1-msec effect when a neutral item was cued and a 2-msec effect when the target was cued. These differences are reflected in the significant interactions of task, cue location, and task and cue location with orientation compatibility, as well as the significant interaction of cue location and orientation compatibility in the error analysis. In the error analysis, the significant task $\times$ cue location $\times$ orientation compatibility interaction goes counter to a similar interaction in RTs, in that the largest compatibility effects are seen in the letter task when the foil is cued. However, this is probably a ceiling effect, since overall error rates are much lower in the orientation task, leaving little room for compatibility effects to appear.

Finally, the interaction of task, letter compatibility, and orientation compatibility in RTs reflects the fact that letter compatibility is effective primarily in the letter task, whereas orientation compatibility is primarily effective in the orientation task. On the letter task, there was an 8-msec effect of letter compatibility but only a 4-msec effect of orientation compatibility. On the orientation task, there was a 2-msec effect of letter compatibility but a 22-msec effect of orientation compatibility.

\section{Detailed Analysis of Experiment 2}

In Experiment 2, five factors were manipulated orthogonally: task (letter vs. orientation), cue location (foil, neutral, or target), prepared task (letter or orientation), compatibility of the letter identity of the foil with the target (compatible or incompatible), and compatibility of the orientation of the foil with the target (compatible or incompatible). Mean RTs and error rates for each cell in this design are presented in Table A3. ANOVAs were performed on both RTs and error rates. The results of this ANOVA appear in Table A4.

The effects of both task and cue location were again significant (although, unlike in Experiment 1, their interaction was only marginally significant in RTs and nonsignificant in error rates). The participants were faster $(604 \mathrm{msec})$ and more accurate $(2.2 \%$ errors $)$ on the orientation task than on the letter task $(647 \mathrm{msec}$; $3.7 \%$ errors). The task $\times$ preparation interaction was also significant (showing that the participants did prepare for each task): On average, the participants took $597 \mathrm{msec}$ and made $3.2 \%$ errors on the letter task when it was prepared for but took $699 \mathrm{msec}$ and made $4.3 \%$ errors when they prepared for the orientation task. Similarly, the participants took $562 \mathrm{msec}$ and made $1.8 \%$ errors on the orientation task when it was prepared for but took $647 \mathrm{msec}$ and made $2.6 \%$ errors when they prepared for the letter task.

The effect of compatibility with the letter identity of the foil was significant in RTs, but, as in Experiment 1, this effect must be looked at in the context of its significant interaction with task and cue location. There was a 25 -msec effect of letter compatibility in the letter task when the foil was cued. In all other conditions, the effect of letter compatibility varied between -7 and $12 \mathrm{msec}$.

Similarly, the effect of compatibility with the orientation of the foil must be looked at in the context of a significant interaction with task and cue location. There was a $50-\mathrm{msec}$ effect of orientation compatibility in the orientation task when the foil was cued. In all the other conditions, the effect of orientation compatibility varied between -3 and 13 msec.

Interestingly, there was a $12-\mathrm{msec}$ effect of letter compatibility in the orientation task when the target was cued and a similar 13-msec effect of orientation compatibility in the letter task when the target was cued. We have no explanation for why cuing the target would result in an increased compatibility effect for the nontask dimension. No similar effect was found in Experiment 1 or in Remington and Folk's (2001) similar experiments.

Table A3

Mean Response Times (RTs, in Milliseconds) and Error Rates (\%E) for Experiment 2

\begin{tabular}{|c|c|c|c|c|c|c|c|c|c|c|}
\hline \multirow[b]{3}{*}{ Task } & \multirow[b]{3}{*}{ Cue } & \multirow[b]{3}{*}{ Prepared } & \multicolumn{8}{|c|}{ Compatibility by Dimension (Letter/Orientation) } \\
\hline & & & \multicolumn{2}{|c|}{$\mathrm{Com} / \mathrm{Com}$} & \multicolumn{2}{|c|}{$\mathrm{Com} / \mathrm{Inc}$} & \multicolumn{2}{|c|}{ Inc/Com } & \multicolumn{2}{|c|}{ Inc/Inc } \\
\hline & & & RT & $\% \mathrm{E}$ & $\mathrm{RT}$ & $\% \mathrm{E}$ & RT & $\% \mathrm{E}$ & $\mathrm{RT}$ & $\% \mathrm{E}$ \\
\hline \multirow[t]{6}{*}{ Letter } & Target & Letter & 538 & 2.3 & 562 & 2.1 & 550 & 1.6 & 555 & 2.1 \\
\hline & & Orientation & 653 & 3.2 & 667 & 3.8 & 639 & 3.7 & 649 & 5.5 \\
\hline & Foil & Letter & 626 & 2.4 & 622 & 5.0 & 639 & 3.1 & 653 & 7.2 \\
\hline & & Orientation & 722 & 2.3 & 729 & 7.7 & 754 & 3.8 & 754 & 3.7 \\
\hline & Neutral & Letter & 603 & 3.0 & 594 & 3.2 & 615 & 2.6 & 610 & 3.7 \\
\hline & & Orientation & 713 & 6.7 & 712 & 4.7 & 692 & 2.4 & 697 & 4.1 \\
\hline \multirow[t]{6}{*}{ Orientation } & Target & Letter & 600 & 0.7 & 610 & 1.4 & 616 & 3.2 & 617 & 0.4 \\
\hline & & Orientation & 513 & 1.0 & 510 & 1.2 & 520 & 1.3 & 528 & 0.2 \\
\hline & Foil & Letter & 654 & 0.0 & 695 & 4.0 & 645 & 2.1 & 715 & 6.3 \\
\hline & & Orientation & 571 & 0.9 & 618 & 3.2 & 570 & 2.0 & 611 & 4.4 \\
\hline & Neutral & Letter & 651 & 1.6 & 645 & 2.9 & 653 & 3.3 & 668 & 5.4 \\
\hline & & Orientation & 568 & 2.2 & 582 & 2.0 & 575 & 1.2 & 583 & 1.5 \\
\hline
\end{tabular}

Note-Com, compatible; Inc, incompatible. 
APPENDIX (Continued)

Table A4

ANOVA Results for Experiment 2

\begin{tabular}{|c|c|c|c|c|c|}
\hline \multirow[b]{2}{*}{ Source } & \multirow[b]{2}{*}{$d f$} & \multicolumn{2}{|c|}{ Response Times } & \multicolumn{2}{|c|}{ Errors } \\
\hline & & $F$ & $p$ & $F$ & $p$ \\
\hline Task & 1,45 & 10.05 & .003 & 16.18 & .000 \\
\hline Cue & 2,90 & 183.42 & .000 & 7.61 & .001 \\
\hline Task $\times$ cue & 2,90 & 2.81 & .066 & 0.40 & .671 \\
\hline Preparation & 1,45 & 0.58 & .450 & 0.14 & .708 \\
\hline Task $\times$ preparation & 1,45 & 194.92 & .000 & 9.08 & .004 \\
\hline Cue $\times$ preparation & 2,90 & 0.49 & .617 & 1.92 & .153 \\
\hline Task $\times$ cue $\times$ preparation & 2,90 & 1.24 & .296 & 1.92 & .153 \\
\hline Letter & 1,45 & 9.53 & .003 & 1.08 & .304 \\
\hline Task $\times$ letter & 1,45 & 0.08 & .778 & 2.87 & .097 \\
\hline Cue $\times$ letter & 2,90 & 1.81 & .169 & 1.33 & .270 \\
\hline Task $\times$ cue $\times$ letter & 2,90 & 7.46 & .001 & 1.28 & .284 \\
\hline Preparation $\times$ letter & 1,45 & 2.73 & .106 & 4.30 & .044 \\
\hline Task $\times$ preparation $\times$ letter & 1,45 & 0.78 & .382 & 0.20 & .660 \\
\hline Cue $\times$ preparation $\times$ letter & 2,90 & 1.13 & .328 & 1.93 & .151 \\
\hline Task $\times$ cue $\times$ preparation $\times$ letter & 2,90 & 1.23 & .296 & 1.05 & .354 \\
\hline Orientation & 1,45 & 21.33 & .000 & 15.62 & .000 \\
\hline Task $\times$ orientation & 1,45 & 11.15 & .002 & 0.11 & .737 \\
\hline Cue $\times$ orientation & 2,90 & 6.88 & .002 & 9.77 & .000 \\
\hline Task $\times$ cue $\times$ orientation & 2,90 & 11.61 & .000 & 1.54 & .219 \\
\hline Preparation $\times$ orientation & 1,45 & 0.01 & .918 & 0.86 & .360 \\
\hline Task $\times$ preparation $\times$ orientation & 1,45 & 0.11 & .743 & 0.43 & .514 \\
\hline Cue $\times$ preparation $\times$ orientation & 2,90 & 0.71 & .493 & 1.49 & .231 \\
\hline Task $\times$ cue $\times$ preparation $\times$ orientation & 2,90 & 0.10 & .908 & 0.02 & .984 \\
\hline Letter $\times$ orientation & 1,45 & 0.28 & .597 & 0.00 & .969 \\
\hline Task $\times$ letter $\times$ orientation & 1,45 & 0.34 & .564 & 0.50 & .485 \\
\hline Cue $\times$ letter $\times$ orientation & 2,90 & 0.64 & .530 & 1.79 & .173 \\
\hline Task $\times$ cue $\times$ letter $\times$ orientation & 2,90 & 0.10 & .905 & 2.02 & .138 \\
\hline Preparation $\times$ letter $\times$ orientation & 1,45 & 0.73 & .398 & 0.05 & .820 \\
\hline Task $\times$ preparation $\times$ letter $\times$ orientation & 1,45 & 0.31 & .580 & 0.50 & .485 \\
\hline Cue $\times$ preparation $\times$ letter $\times$ orientation & 2,90 & 1.99 & .143 & 1.95 & .149 \\
\hline Task $\times$ cue $\times$ preparation $\times$ letter $\times$ orientation & 2,90 & 0.20 & .823 & 1.35 & .264 \\
\hline
\end{tabular}

\section{Detailed Analysis of Experiment 3}

In Experiment 3, four factors were manipulated orthogonally: task (letter vs. orientation), prepared task (letter or orientation), compatibility of the letter identity of the foil with the target (compatible or incompatible), and compatibility of the orientation of the foil with the target (compatible or incompatible). Mean RTs and error rates for each cell in this design are presented in Table A5. ANOVAs were performed on both RTs and error rates. The results of this ANOVA appear in Table A6.

The effect of task was again significant. The participants were faster $(585 \mathrm{msec})$ and more accurate $(2.9 \%$ errors) on the orientation task than on the letter task ( $650 \mathrm{msec} ; 5.2 \%$ errors). Responses when the task was prepared for were again faster and more accurate than those for the unprepared for task. The participants, on average, took $609 \mathrm{msec}$ to respond and made 3.6\% errors in the letter task when they prepared for the letter task but took $691 \mathrm{msec}$ and made $6.7 \%$ errors when they prepared for the orientation task. Similarly, the participants took $547 \mathrm{msec}$ and made $2.6 \%$ errors in the orientation task when they prepared for the orientation task but took $622 \mathrm{msec}$ and made $3.3 \%$ errors when they prepared for the letter task.

Unlike in the other experiments reported here, in this experiment there are hints of an interaction between compatibility and preparation. The effect of compatibility with the letter identity of the foil was significant in the error analysis (3.5\% errors when the letter was compatible vs. $4.6 \%$ errors when it was not) and approached

Table A5

Mean Response Times (RTs, in Milliseconds) and Error Rates (\%E) for Experiment 3

\begin{tabular}{|c|c|c|c|c|c|c|c|c|c|}
\hline \multirow[b]{3}{*}{ Task } & \multirow[b]{3}{*}{ Prepared } & \multicolumn{8}{|c|}{ Compatibility by Dimension (Letter/Orientation) } \\
\hline & & \multicolumn{2}{|c|}{ Com/Com } & \multicolumn{2}{|c|}{ Com/Inc } & \multicolumn{2}{|c|}{ Inc/Com } & \multicolumn{2}{|c|}{ Inc/Inc } \\
\hline & & RT & $\% \mathrm{E}$ & RT & $\% \mathrm{E}$ & RT & $\% \mathrm{E}$ & RT & $\% \mathrm{E}$ \\
\hline \multirow[t]{2}{*}{ Letter } & Letter & 592 & 2. & 606 & 3. & 61 & 3.2 & 62 & 4.7 \\
\hline & Orientation & 690 & 3.3 & 698 & 8.1 & 678 & 6.3 & 699 & 8.9 \\
\hline \multirow{2}{*}{ Orientation } & Letter & 614 & 2.2 & 634 & 3.1 & 602 & 2.8 & 639 & 5.2 \\
\hline & Orientation & 521 & 1.7 & 562 & 3.0 & 530 & 1.2 & 575 & 4.3 \\
\hline
\end{tabular}

Note-Com, compatible; Inc, incompatible. 
APPENDIX (Continued)

Table A6

ANOVA Results for Experiment 3

\begin{tabular}{|c|c|c|c|c|c|}
\hline \multirow[b]{2}{*}{ Source } & \multirow[b]{2}{*}{$d f$} & \multicolumn{2}{|c|}{ Response Times } & \multicolumn{2}{|c|}{ Errors } \\
\hline & & $F$ & $p$ & $F$ & $p$ \\
\hline Task & 1,37 & 26.47 & .000 & 16.34 & .000 \\
\hline Preparation & 1,37 & 0.07 & .795 & 5.62 & .023 \\
\hline Task $\times$ preparation & 1,37 & 171.96 & .000 & 19.64 & .000 \\
\hline Letter & 1,37 & 4.03 & .052 & 5.22 & .028 \\
\hline Task $\times$ letter & 1,37 & 0.60 & .444 & 0.33 & .568 \\
\hline Preparation $\times$ letter & 1,37 & 1.62 & .210 & 0.03 & .863 \\
\hline Task $\times$ preparation $\times$ letter & 1,37 & 19.02 & .000 & 2.00 & .166 \\
\hline Orientation & 1,37 & 77.77 & .000 & 25.54 & .000 \\
\hline Task $\times$ orientation & 1,37 & 16.25 & .000 & 0.77 & .385 \\
\hline Preparation $\times$ orientation & 1,37 & 1.96 & .170 & 4.36 & .044 \\
\hline Task $\times$ preparation $\times$ orientation & 1,37 & 1.78 & .190 & 2.61 & .115 \\
\hline Letter $\times$ orientation & 1,37 & 3.10 & .087 & 0.35 & .558 \\
\hline Task $\times$ letter $\times$ orientation & 1,37 & 0.26 & .613 & 3.97 & .054 \\
\hline Preparation $\times$ letter $\times$ orientation & 1,37 & 0.05 & .825 & 0.82 & .372 \\
\hline Task $\times$ preparation $\times$ letter $\times$ orientation & 1,37 & 2.32 & .136 & 1.18 & .285 \\
\hline
\end{tabular}

significance in the RT analysis (615 msec when the letter was compatible vs. $620 \mathrm{msec}$ when it was not). However, the effect on RTs was modulated by task and preparation. Compatibility with the letter identity of the foil had a 21-msec effect when the letter task was both prepared for and executed and had an 11-msec effect when the orientation task was both prepared for and executed but had little effect $(-3 \mathrm{msec})$ when the prepared for task was not actually executed. Note that, although preparation clearly modulated the effect of letter compatibility, it is not the effect one might expect if one believed that preparation caused some sort of selective filtering of that dimension. Rather than an increase in the effect of letter compatibility when the participants prepared for the letter task, we see an increase in the effect of letter compatibility when the prepared for task was actually executed. Thus, it appears to be due to the participants doing a sort of mental reset when they receive the unexpected task.

The effect of compatibility with the orientation of the foil was significant in both the RT and error analyses. The effect on RTs was modulated by task. There was a 36-msec effect of orientation compatibility when the orientation task was executed but only a $13-\mathrm{msec}$ effect when the letter task was executed. The effect on errors was modulated by preparation. When the participants prepared for the orientation task, they made $3.1 \%$ errors when the orientation of the foil was compatible but $6.1 \%$ errors when it was incompatible. When the participants prepared for the letter task, they made $2.7 \%$ errors when the orientation of the foil was compatible and $4.2 \%$ errors when it was incompatible.

\section{Detailed Analysis of Experiment 4}

Data from conditions in which the foil was physically identical to the target were discarded. Experiment 4 had a design different from that of the earlier experiments. In particular, the foil had either an orientation or a letter identity but not both. As a result, the dimension and the compatibility of the foil become separate factors. Thus, in Experiment 4, four factors were manipulated orthogonally: task (letter vs. orientation), prepared task (letter or orientation), dimension of the foil (letter or orientation), and compatibility of the foil with the target (compatible or incompatible). Mean RTs and error rates for each cell in this design are presented in Table A7. ANOVAs were performed on both RTs and error rates. The results of this ANOVA appear in Table A8.

The effect of task was again significant; however, in this experiment, the participants were faster (664 msec) and more accurate ( $2.8 \%$ errors) in the letter task than in the orientation task ( $677 \mathrm{msec} ; 4.0 \%$ errors). There was a significant effect of the prepared for task on errors and a marginally significant effect on RTs; however, this effect must be viewed in the context of the significant interaction of task and prepared for task. The participants, on average, took $649 \mathrm{msec}$ to respond and made $2.7 \%$ errors in the letter task when they prepared for the letter

Table A7

Mean Response Times (RTs, in Milliseconds) and Error Rates (\%E) for Experiment 4

\begin{tabular}{|c|c|c|c|c|c|c|c|c|c|}
\hline \multirow[b]{3}{*}{ Task } & \multirow[b]{3}{*}{ Prepared } & \multicolumn{4}{|c|}{ Letter Foil } & \multicolumn{4}{|c|}{ Orientation Foil } \\
\hline & & \multicolumn{2}{|c|}{ Compatible } & \multicolumn{2}{|c|}{ Incompatible } & \multicolumn{2}{|c|}{ Compatible } & \multicolumn{2}{|c|}{ Incompatible } \\
\hline & & RT & $\% \mathrm{E}$ & RT & $\% \mathrm{E}$ & RT & $\% \mathrm{E}$ & RT & $\% \mathrm{E}$ \\
\hline \multirow[t]{2}{*}{ Letter } & Letter & 633 & 2.4 & 648 & 2.7 & 650 & 3.0 & 665 & 2.8 \\
\hline & Orientation & 670 & 2.2 & 662 & 1.6 & 692 & 3.4 & 692 & 4.2 \\
\hline \multirow[t]{2}{*}{ Orientation } & Letter & 728 & 6.3 & 716 & 4.8 & 666 & 4.5 & 686 & 3.9 \\
\hline & Orientation & 663 & 2.9 & 668 & 3.6 & 636 & 1.9 & 653 & 4.4 \\
\hline
\end{tabular}


APPENDIX (Continued)

Table A8

ANOVA Results for Experiment 4

\begin{tabular}{|c|c|c|c|c|c|}
\hline \multirow[b]{2}{*}{ Source } & \multirow[b]{2}{*}{$d f$} & \multicolumn{2}{|c|}{ Response Times } & \multicolumn{2}{|c|}{ Errors } \\
\hline & & $F$ & $p$ & $F$ & $p$ \\
\hline Task & 1,41 & 5.39 & .025 & 9.63 & .003 \\
\hline Preparation & 1,41 & 3.36 & .074 & 6.12 & .018 \\
\hline Task $\times$ preparation & 1,41 & 90.66 & .000 & 7.69 & .008 \\
\hline Foil & 1,41 & 5.65 & .022 & 0.42 & .519 \\
\hline Task $\times$ foil & 1,41 & 66.97 & .000 & 6.16 & .017 \\
\hline Preparation $\times$ foil & 1,41 & 9.47 & .004 & 5.93 & .019 \\
\hline Task $\times$ preparation $\times$ foil & 1,41 & 2.78 & .103 & 0.04 & .835 \\
\hline Congruence & 1,41 & 7.07 & .011 & 0.17 & .680 \\
\hline Task $\times$ congruence & 1,41 & 0.16 & .694 & 0.25 & .623 \\
\hline Preparation $\times$ congruence & 1,41 & 0.17 & .684 & 2.73 & .106 \\
\hline Task $\times$ preparation $\times$ congruence & 1,41 & 3.91 & .055 & 13.07 & .001 \\
\hline Foil $\times$ congruence & 1,41 & 3.05 & .088 & 0.12 & .736 \\
\hline Task $\times$ foil $\times$ congruence & 1,41 & 5.30 & .026 & 4.73 & .036 \\
\hline Preparation $\times$ foil $\times$ congruence & 1,41 & 2.58 & .116 & 0.00 & .965 \\
\hline Task $\times$ preparation $\times$ foil $\times$ congruence & 1,41 & 0.96 & .332 & 0.67 & .417 \\
\hline
\end{tabular}

task but 679 msec with $2.8 \%$ errors when they prepared for the orientation task. Similarly, the participants took $655 \mathrm{msec}$ and made $3.2 \%$ errors in the orientation task when they prepared for the orientation task but took $699 \mathrm{msec}$ and made $4.8 \%$ errors when they prepared for the letter task.

Interestingly, the foils themselves appeared to affect task preparation. Independently of whether the foil was congruent or incongruent, the participants were faster and more accurate when the foil dimension matched the executed task. The participants, on average, took $653 \mathrm{msec}$ to respond and made $2.2 \%$ errors in the letter task when the foil was a letter but took $674 \mathrm{msec}$ and made $3.3 \%$ errors when it was an oriented box. Similarly, the participants took $661 \mathrm{msec}$ and made $3.7 \%$ errors in the orientation task when the foil was an oriented box but took $693 \mathrm{msec}$ and made $4.4 \%$ errors when the foil was a letter.

Compatibility of the foil was significant for RTs; however, this effect must be looked at in the context of two higher order interactions. First, the task $\times$ preparation $\times$ compatibility interaction was significant in the error analysis and marginally significant in RTs. Echoing Experiment 3, compatibility effects were stronger when the task that was prepared for was executed, independently of whether the participants prepared for the foil dimension. In the RT analysis, when the letter task was both prepared for and executed, there was a 15-msec compatibility effect, but when the orientation task was prepared for, the compatibility effect was only $4 \mathrm{msec}$. Similarly, when the orientation task was both prepared for and executed, there was an 11-msec compatibility effect, but when the letter task was prepared for, the compatibility effect was only $4 \mathrm{msec}$. In the error analysis, there was no compatibility effect when the participants executed the letter identification task, regardless of expectation. However, when the participants executed the orientation task, there was a $1.6 \%$ compatibility effect when they prepared for the orientation task but a $-1.1 \%$ compatibility effect when they prepared for the letter task.

Finally, the interaction of compatibility with task and foil dimension was significant in both the RT and error analyses. Compatibility effects were larger when the foil dimension was the same as the dimension of the executed task. When the participants executed the letter task, there was an $11-\mathrm{msec}$ and a $0.4 \%$ compatibility effect with letter foils but only an $8-\mathrm{msec}$ and a $-0.5 \%$ compatibility effect with orientation foils. Similarly, when the participants executed the orientation task, there was a $19-\mathrm{msec}$ and a $1.0 \%$ compatibility effect with orientation foils but only a $-4-\mathrm{msec}$ and a $-0.4 \%$ compatibility effect with letter foils.

Importantly, the interaction of compatibility with foil dimension and preparation was not significant in either the RT or the error analysis. Thus, preparation for a particular dimension did not influence compatibility effects.

(Manuscript received April 14, 2005;

revision accepted for publication February 19, 2009.) 